\title{
Optimized LTE Cell Planning with Varying Spatial and Temporal User Densities
}

\author{
Hakim Ghazzai, Member, IEEE, Elias Yaacoub, Senior Member, IEEE, Mohamed-Slim Alouini, Fellow, \\ IEEE, Zaher Dawy, Senior Member, IEEE, and Adnan Abu-Dayya, Senior Member, IEEE
}

\begin{abstract}
Base station deployment in cellular networks is one of the fundamental problems in network design. This paper proposes a novel method for the cell planning problem for the fourth generation (4G) cellular networks using meta-heuristic algorithms. In this approach, we aim to satisfy both cell coverage and capacity constraints simultaneously by formulating an optimization problem that captures practical planning aspects. The starting point of the planning process is defined through a dimensioning exercise that captures both coverage and capacity constraints. Afterwards, we implement a meta-heuristic algorithm based on swarm intelligence (e.g., particle swarm optimization or the recently-proposed grey wolf optimizer) to find suboptimal base station locations that satisfy both problem constraints in the area of interest which can be divided into several subareas with different spatial user densities. Subsequently, an iterative approach is executed to eliminate eventual redundant base stations. We also perform Monte Carlo simulations to study the performance of the proposed scheme and compute the average number of users in outage. Next, the problems of green planning with regards to temporal traffic variation and planning with location constraints due to tight limits on electromagnetic radiations are addressed, using the proposed method. Finally, in our simulation results, we apply our proposed approach for different scenarios with different subareas and user distributions and show that the desired network quality of service targets are always reached even for large-scale problems.
\end{abstract}

Index Terms-LTE Cellular Network Planning, Coverage and Cell Capacity Dimensioning, Green Planning, Electromagnetic Radiation Exposure, Meta-Heuristic Algorithms.

\section{INTRODUCTION}

Network planning is a classical but important problem in designing cellular networks. It mostly includes the planning of base stations (BSs) by optimizing their locations and configurations in order to provide full coverage of the service area with respect to the traffic requirements, available capabilities, and the desired quality of service (QoS) [2]-[4]. Under these constraints, the main objective is to reduce the total cost

Copyright (c) 2015 IEEE. Personal use of this material is permitted. However, permission to use this material for any other purposes must be obtained from the IEEE by sending a request to pubs-permissions@iee.org.

A part of this work has been presented at the Vehicular Technology Conference (VTC-Fall 2014), Vancouver, Canada [1].

The work was made possible by NPRP grant \#4-353-2-130 from the Qatar National Research Fund (a member of Qatar Foundation). The statements made herein are solely the responsibility of the authors.

Hakim Ghazzai and Mohamed-Slim Alouini are with the Electrical Engineering Program, Computer, Electrical and Mathematical Sciences and Engineering (CEMSE) Division, King Abdullah University of Science and Technology (KAUST), Thuwal, Makkah Province, Saudi Arabia. (E-mails: \{hakim.ghazzai, slim.alouini\}@kaust.edu.sa).

Elias Yaacoub is with the Strategic Decision Group (SDG), Beirut, Lebanon. (E-mail: eliasy@ieee.org).

Zaher Dawy is with the Electrical and Computer Engineering Department, American University of Beirut, Beirut, Lebanon. (E-mail: zd03@aub.edu.lb)

Adnan Abu-Dayya is with Qatar Mobility Innovations Center (QMIC), Qatar Science \& Technology Park, Doha, Qatar. (E-mail: adnan@qmic.com) for deploying and expanding the cellular system. Indeed, this fundamental planning task is a result of optimization problems to determine the number and the locations of the BSs in order to meet coverage and capacity requirements. The problem requires the knowledge of several parameters as inputs related to the employed technology and the geographical distribution of the traffic demand which increases its complexity and makes the optimal problem solution difficult if not impossible to reach.

Several work have been proposed to study the deployment of BSs. Most of these studies were based on heuristic approaches to solve this NP-Hard problem [5]. For instance, [6] and [7] employed the tabu search and the genetic algorithm, respectively, to preform cell planning for code division multiple access systems. Few previous works dealt with optimizing the BS locations for fourth generation long term evolution (4G-LTE). The authors in [8] proposed a mixed integer programming model with the use of the method of the Pareto front and multi-objective tabu search to optimize cell planning. Another approach, presented in [9], proposed to determine the BS locations based on stochastic models such as the Poisson point process and considering the average squared error of the coverage probability as goodness criterion. In [10], the authors proposed an algorithm for joint uplink/downlink universal mobile telecommunications system (UMTS) radio planning with the objective of minimizing total power consumption in the network. The problem is subdivided into two components that are executed successively: First, the authors aimed to find the optimal positions of a fixed number of UMTS BSs in the area of interest. The optimal locations of BSs are obtained by solving an optimization problem that aims to minimize the total downlink power expenditure and, at the same time, the uplink outage that depends on the power capabilities of mobile stations (MSs) under different constraints that maintain an acceptable QoS and satisfy the power budget. As a second step, the authors proposed an algorithm to select the minimal cardinality set of BSs with fixed locations. In [11], the authors started by placing randomly a high number of BSs in the area of interest. Then, they employed an iterative algorithm based on user snapshot studies to eliminate redundant BSs. Similarly, the authors in [12] proposed a heuristic algorithm to meet green objectives by selecting BSs from a predefined set of candidates. The proposed solutions did not present a general solution as the elimination step depends on the user realization and the selected BS locations may vary from a realization to another. Moreover, most of the proposed schemes are not adapted to a given area divided into several subareas with varied user densities. Indeed, it is useful to consider an area 
of interest consisting of multiple subareas with different user densities, e.g., if it comprises a shopping or business district located near a residential area, where the characteristics of each of these subareas should be considered in the planning process instead of the traditional uniform user densities.

In this paper, we propose an optimized LTE-Advanced radio planning method by formulating a combinatorial optimization problem that aims to find the optimal locations of the minimum number of BSs to be deployed in a given area of interest while respecting two important constraints in the planning process: the area coverage constraint and the cell capacity constraint. We propose to exploit swarm intelligence in order to solve the planning optimization problem for varied spatial and temporal user densities. After evaluating the link power budget and estimating the number of BSs needed to be deployed and their radius using a radio propagation model, we employ metaheuristic algorithms based on swarm intelligence to find their suboptimal locations. In our study, we propose to employ the particle optimization algorithm (PSO) [13] in addition to the recently proposed grey wolf optimizer (GWO) [14]. Then, we compare their performances and their speed of convergence with the probabilistic metaheuristic algorithm: The simulated annealing (SA) [15]. Finally, we eliminate redundant BSs using a low complexity iterative algorithm. This is performed by dividing the area of interest into several subareas characterized by different user densities and taking into account both the uplink (UL) and downlink (DL) directions, LTE resource allocation, and intercell interference. Afterwards, we apply the proposed method to ensure a proactive green planning that takes into consideration the temporal traffic variation. In this approach, based on traffic statistics, the mobile operator can identify the BSs to be turned off during night or low traffic period from the planning stage, in order to optimize the energy efficiency during post-deployment network operation. Moreover, we adapt the proposed method to solve the planning problem with location constraints whereby the placement of BSs is not allowed in some regions of the area of interest, e.g., due to private property or electromagnetic radiation constraints [10]. In addition, we apply our proposed planning approach to the case where femtocells, that offload an amount of the traffic from cellular networks, are deployed in the area of interest and to the case of LTE heterogeneous (hetnet) networks where macrocell BSs and small cell BSs co-exist. The performance evaluation of the proposed method is performed using Monte Carlo simulations that measure the average percentage of users in outage.

Compared to previous proposed approaches, our planning method for LTE-Advanced is first based on an estimation of the total number of BSs using the dimensioning phase where all the system parameters are taken into account. Second, it is not limited to a finite set of predefined BS locations, allows the operator to optimize both cell capacity and coverage constraints simultaneously, and tries to find the minimal number of BSs to be deployed. Third, it considers more realistic scenarios depending on user densities in neighboring areas for which a network is being planned instead of focusing on the traditional single uniform region. In addition, it simultaneously encloses the spatial and temporal user density variation, the location constraint problem, and the identification of the BSs that would be turned off since the planning stage. Finally, our approach takes resource allocation into account and is applicable with any radio resource management algorithm.

The rest of this paper is organized as follows. Section II presents the dimensioning phase and the formulated optimization problem. Section III describes the proposed algorithm based on the meta-heuristic algorithms. In Section IV, the optimization problem is reformulated to deal with the green planning and electromagnetic radiation exposure problem. The performance evaluation method is presented in Section V. Next, simulation results are given in Section VI. Finally, Section VII concludes the paper.

\section{System Parameters and Problem Formulation}

In cellular networks, coverage and capacity should be considered simultaneously in order to avoid limited range of coverage [16]. In this section, we formulate an optimization problem that fulfills the coverage and data rate requirements by deploying the minimum number of BSs. Indeed, network operators need to place their BSs in a manner that allows each user in the service area to communicate with at least one of these BSs. On the other hand, mobile operators have to meet their user QoSs by providing the needed throughput for the service operation. Thus, the objective is to find the vectors $\boldsymbol{x}$ and $\boldsymbol{y}$ with dimension up to $N_{\mathrm{BS}}$ (i.e., BS positions in the Cartesian coordinate system) that satisfy the planning constraints where $N_{\mathrm{BS}}$ is the initial number of candidate BSs to deploy. We assume that each BS is equipped with $N_{\mathrm{S}}$ sector antennas as it is shown in Fig. 1. The antenna gain is set to 18 $\mathrm{dBi}$ and its pattern is given in (36) in Section V. The aim is to serve an area with a total surface denoted $\mathcal{A}_{T}$ and expressed in kilometer-square $\left(\mathrm{km}^{2}\right)$. The area can be subdivided into $N_{\text {Area }}$ subareas. Each subarea $i$ is characterized by its surface $\mathcal{A}(i)$ (i.e., $\sum_{i=1}^{N_{\text {Area }}} \mathcal{A}(i)=\mathcal{A}_{T}$ ) and a particular user density function $\mathcal{D}_{i}$. For instance, the density could be a uniform distribution with a given user density per $\mathrm{km}^{2}$ or a normal (Gaussian) distribution corresponding to concentrated users in a hotspot region and then the density is reduced as we move away from the center, etc. A dimensioning phase is initially performed to find the radius $R_{\mathrm{BS}}$ of cells and an estimate of $N_{\mathrm{BS}}$ base stations as a function of the given coverage and capacity constraints. In addition, it gives an estimate of the number of users that can be served simultaneously by a BS that we denote by $N_{U_{\mathrm{BS}}}$.

\section{A. Initial Dimensioning Phase}

Coverage dimensioning: This phase begins by computing the radio link budget [17]. The link budget estimates the maximum allowed signal attenuation between the mobile and the BS antenna. An example of radio link budget of UL and DL directions for LTE networks is given in Table I in Section VI. The radio link budget parameters are selected from [17], [18]. In LTE, orthogonal frequency division multiple access (OFDMA) is the access scheme for the DL while single carrier frequency division multiple access (SCFDMA) is used in UL. The available spectrum is divided into resource blocks (RB) consisting of 12 adjacent subcarriers. The DL and UL 


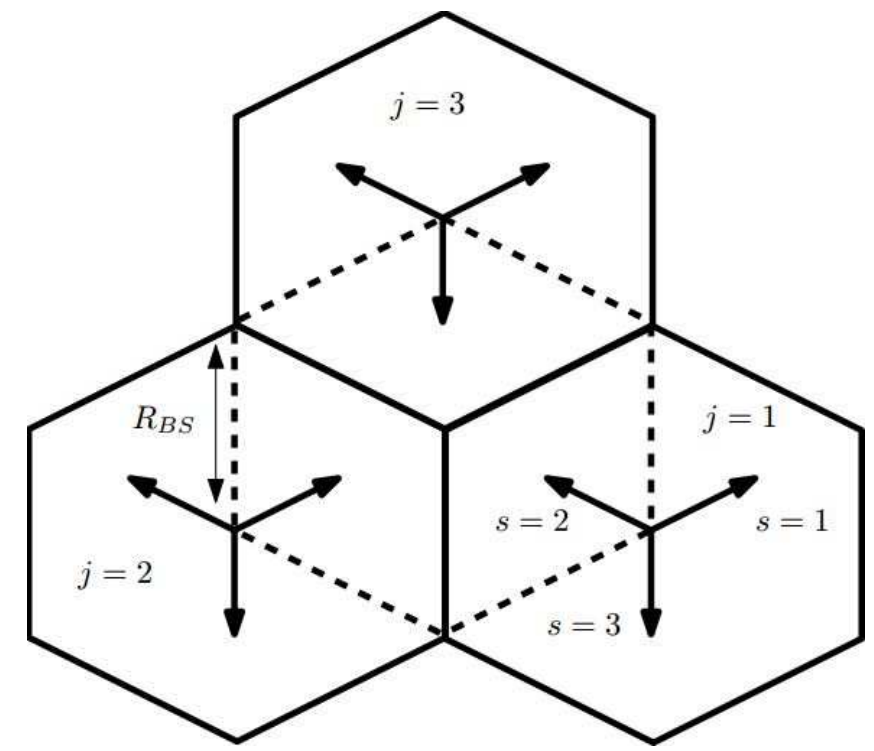

Fig. 1: Three sector antenna base stations serving an hexagonal cell.

maximum allowed path losses (MAPLs) can be compared together to determine whether UL or DL coverage is limited and thus determine the cell range accordingly. The cell ranges are calculated using the COST-231-HATA propagation model, which computes the path loss, denoted PL [19]. Thus, we can determine the cell radius $R_{\mathrm{BS}}=\left(\left.d\right|_{\mathrm{PL}=\mathrm{MAPL}}\right)$ where MAPL is computed after elaborating the UL and DL budgets. Once $R_{\mathrm{BS}}$ is fixed, the number of BSs needed to cover the area of interest $N_{\mathrm{BS}}^{\mathrm{Cov}}$ is given as follows:

$$
N_{\mathrm{BS}}^{\mathrm{Cov}}=\left\lceil\frac{\mathcal{A}_{T}}{S_{\text {Cell }}}\right\rceil,
$$

where the symbol $\lceil$.$\rceil denotes ceiling function and S_{\text {Cell }}$ is the surface of the cell with radius $R_{\mathrm{BSS}}$. For instance, $S_{\text {Cell }}=\pi\left(R_{\mathrm{BS}}\right)^{2}$ for a circular cell and $S_{\text {Cell }}=\frac{3 \sqrt{3}}{2}\left(R_{\mathrm{BS}}\right)^{2}$ for a hexagonal cell.

Capacity Dimensioning: The objective of this phase is to determine an estimate of the maximum number of users $N_{U_{\mathrm{BS}}}$ that can be served by one BS simultaneously, then, to find the number of BSs $N_{\mathrm{BS}}^{\mathrm{Cap}}$ required to satisfy the DL user data rate. The DL data rate is only considered in this capacity dimensioning phase as it is the limiting link in terms of capacity provisioning due to the fact that it is usually higher than the UL data rate. We assume that users have a target data rate that they aim to achieve in DL, denoted $R_{\mathrm{th}}^{(\mathrm{DL})}$. Thus, $N_{U_{\mathrm{BS}}}$ is defined as follows:

$$
N_{U_{\mathrm{BS}}}=\left\lfloor\frac{N_{\mathrm{S}} C_{\mathrm{Cell}}^{s}}{R_{\mathrm{th}}^{(\mathrm{DL})}}\right\rfloor,
$$

where the symbol $\lfloor\cdot\rfloor$ denotes the floor function and $C_{\text {Cell }}^{s}$ is the cell capacity per sector antenna which corresponds to the maximum data rate that will be shared between all connected users to a sector antenna $s$ and defined as $C_{\text {Cell }}^{s}=B \times \mathrm{SE}$ where $B$ is the channel bandwidth in $(\mathrm{Hz})$ while $\mathrm{SE}$ is the spectral efficiency of the system in (bits $\backslash \mathrm{s} \backslash \mathrm{Hz}$ ). Thus, $N_{\mathrm{BS}}^{\mathrm{Cap}}$ is no more than the sum of the number of BSs $N_{\mathrm{BS}_{i}}^{\mathrm{Cap}}$ needed for each subarea $i$ as follows:

$$
N_{\mathrm{BS}}^{\mathrm{Cap}}=\sum_{i=1}^{N_{\mathrm{Area}}} N_{\mathrm{BS}_{i}}^{\mathrm{Cap}}
$$

where $N_{\mathrm{BS}_{i}}^{\mathrm{Cap}}=\frac{\mathcal{D}_{i} \mathcal{A}(i)}{N_{U_{\mathrm{BS}}}}, \forall i=1, \cdots, N_{\text {Area }}$, which exactly corresponds to the number of users in subarea $i$ divided by the maximum number of simultaneously served users by a cell.

Finally, the estimated number of BSs needed to cover the whole area and satisfy the data rate requirement in each subarea is given as follows:

$$
N_{\mathrm{BS}}=\max \left(N_{\mathrm{BS}}^{\mathrm{Cov}}, N_{\mathrm{BS}}^{\mathrm{Cap}}\right) \text {. }
$$

\section{B. Optimization Problem Formulation}

The optimization problem objective is to find the optimal BS locations $\left(\boldsymbol{x}^{*}, \boldsymbol{y}^{*}\right)$ that satisfy the cell capacity constraint per subarea and the total coverage constraint.

Cell capacity constraint: We associate to each triplet (sector $s$, BS $j$, subarea $i)$ the parameter $\rho_{s, i, j},\left(0 \leq \rho_{s, i, j} \leq 1, \forall s=\right.$ $1, \cdots, N_{\mathrm{S}}, \forall i=1, \cdots, N_{\text {Area }}$ and $\left.\forall j=1, \cdots, N_{\mathrm{BS}}\right)$ to measure the presence of BS $j$ in subarea $i$ as follows:

$$
\rho_{s, i, j}\left(x_{j}, y_{j}\right)=\frac{a_{s, i, j}\left(x_{j}, y_{j}\right)}{A_{s, j}},
$$

where $a_{s, i, j}\left(x_{j}, y_{j}\right)$ is the surface covered by sector $s$ of BS $j$ having as coordinates $\left(x_{j}, y_{j}\right)$ that intersects subarea $i$ in $\mathrm{km}^{2}$ and $A_{s, j}$ is the total area covered by sector $s$ of BS $j$. Thus, the average number of users that can be served by a sector antenna $s$ of BS $j$ in subarea $i$ is $\frac{N_{U_{\mathrm{BS}}}}{N_{\mathrm{S}}} \rho_{s, i, j}\left(x_{j}, y_{j}\right)$ since each sector antenna can serve, in total, $\frac{N_{U_{\mathrm{BS}}}}{N_{\mathrm{S}}}$ users. Hence, if a sector antenna $s$ of BS $j$ is totally included in the subarea $i$, then, $\rho_{s, i, j}\left(x_{j}, y_{j}\right)=1$. If it is partially included in the subarea $i, \rho_{s, i, j}\left(x_{j}, y_{j}\right)$ will be less than 1 . $\rho_{s, i, j}\left(x_{j}, y_{j}\right)=0$ if sector antenna $s$ of BS $j$ and subarea $i$ are disjoint. Therefore, in order to ensure that all users in subarea $i$ are served, the following expression has to be satisfied:

$$
\sum_{j=1}^{N_{\mathrm{BS}}} \sum_{s=1}^{N_{\mathrm{S}}} \frac{N_{U_{\mathrm{BS}}}}{N_{\mathrm{S}}} \rho_{s, i, j}\left(x_{j}, y_{j}\right) \geq \eta \mathcal{D}_{i} \mathcal{A}(i), \forall i=1, \cdots, N_{\text {Area }},
$$

where $\eta$ is a tolerance parameter $(0 \leq \eta \leq 1)$ added to relax the capacity constraint.

Coverage constraint: We propose to distribute uniformly over the area of interest $N_{\text {ref }}$ reference points that have to be covered by at least one of the deployed BSs during the planning phase. If all $N_{\text {ref }}$ points are covered, we can consider that the area is totally covered. Note that increasing $N_{\text {ref }}$ improves the precision of the problem's solution; however, it leads to higher problem complexity. We introduce the binary variable $\gamma_{n}, n=1, \cdots, N_{\text {ref }}$, to denote the state of reference point $n$ as follows:

$\gamma_{n}(\boldsymbol{x}, \boldsymbol{y})= \begin{cases}1 & \text { if point } n \text { is covered by at least one BS } \\ 0 & \text { if point } n \text { is not covered by any BS. }\end{cases}$

A point $n$ is considered covered by one BS if it is covered by one of its sectors. Hence, in order to consider that the area is totally covered, the following equation has to be satisfied:

$$
\sum_{n=1}^{N_{\text {ref }}} \gamma_{n} \leq \tau N_{\text {ref }}
$$


where $\tau$ is a tolerance parameter $0 \leq \tau \leq 1$ added to relax the coverage constraint. Finally, let $\epsilon_{N_{\mathrm{BS}}}$ be a vector that contains the state of each BS as follows:

$$
\epsilon_{j}= \begin{cases}1 & \text { if } \mathrm{BS} j \text { is deployed, } \\ 0 & \text { if } \mathrm{BS} j \text { is redundant. }\end{cases}
$$

Indeed, in the planning phase, some deployed BSs can be redundant and eliminated without affecting the coverage and capacity constraints. Hence, the optimization problem is expressed as follows:

$$
\underset{\boldsymbol{x}, \boldsymbol{y}, \boldsymbol{\epsilon}}{\operatorname{Minimize}} \sum_{j=1}^{N_{\mathrm{BS}}} \epsilon_{j},
$$

Subject to:

$$
\sum_{j=1}^{N_{\mathrm{BS}}} \sum_{s=1}^{N_{\mathrm{S}}} \frac{N_{U_{\mathrm{BS}}}}{N_{\mathrm{S}}} \rho_{s, i, j}\left(x_{j}, y_{j}\right) \geq \eta \mathcal{D}_{i} \mathcal{A}(i), \forall i=1, \cdots, N_{\text {Area }},
$$$$
\sum_{n=1}^{N_{\text {ref }}} \gamma_{n}(\boldsymbol{x}, \boldsymbol{y}) \geq \tau N_{\text {ref }},
$$

Note that the values $\gamma_{n}$ and $\rho_{s, i, j}$ depend directly on the BS locations (i.e., $(\boldsymbol{x}, \boldsymbol{y})$ ).

The planning phase is performed by cellular operators to decide where to deploy base station sites in order to maintain connectivity for long term variation based on average statistics, whereas short term variation, e.g., due to mobility, could be accounted for using different system level techniques such as power control, link adaptation, congestion control, handovers, etc.

\section{Proposed Cell Planning Method}

Generally, the BS location problem is NP-hard [5] and the optimal solution is impossible to reach mainly for largescale problems and when considering an infinite set of possible BS locations. Evolutionary algorithms, such as genetic algorithms [20], simulated annealing [15], PSO [13] and, ant colony optimization [21], are good alternative choices. Therefore, we propose to employ heuristic algorithms that are based on swarm intelligence and can deal with an infinite set of BS combinations unlike other algorithms (e.g., genetic algorithm) that require a finite set of BS combinations to be executed as it is used in [6], [11]. The proposed approach to solve the optimization problem consists of two steps. First, we start by placing the BSs by optimizing their locations $(\boldsymbol{x}, \boldsymbol{y})$ by exploiting the random behavior of the PSO and GWO algorithms, [13], [14]. Then, after fixing the positions of all BSs, we propose to eliminate eventual redundant BSs by dealing with the binary vector $\epsilon$. Due to the following advantages of PSO and GWO compared with the others, we apply them for solving this planning problem: (i) their search processes are simple and easy to implement by manipulating few numerical parameters (e.g., such as the number of particles, inertial weights, and acceleration factors for PSO) (ii) they require low computational cost attained from small number of agents; and (iii) provide a good convergence speed [14], [22]. The PSO algorithm is previously used in literature in different fields while GWO is a recently proposed algorithm which is not yet applied in engineering domain. Thus, we propose to implement both algorithms to solve the planning problem and study their performances.

\section{A. Cell Planning Phase using Meta-Heuristic Algorithms}

1) Particle Swarm Optimization Algorithm for Cell Planning: The PSO idea was introduced in 1995 [13]. It is inspired by swarm intelligence, social behavior, and food searching of a bird flocking and fish schooling. The algorithm is widely used in several wireless communication fields and rapidly developed for its easy implementation and few particles required to be tuned [23]-[25]. In our framework, the algorithm starts by generating $L$ particles $\boldsymbol{W}^{(l)}, l=1, \cdots, L$ of length $2 N_{\mathrm{BS}} \times 1$ to form an initial population $\mathcal{S}$. The vector $\boldsymbol{W}^{(l)}$ contains random BS positions of the particle $l$ within the area of interest as follows:

$$
\boldsymbol{W}^{(l)}=\left[\begin{array}{l}
\boldsymbol{x}^{(l)} \\
\boldsymbol{y}^{(l)}
\end{array}\right]
$$

Then, the PSO computes the following two utilities $U_{1}{ }^{(l)}$ and $U_{2}{ }^{(l)}$ achieved by each particle $l$ :

$$
\begin{aligned}
& U_{1}{ }^{(l)}= \begin{cases}-\sum_{n=1}^{N_{\text {ref }}} \gamma_{n}^{(l)}, & \text { if (11) is satisfied by particle } l, \\
0, & \text { else, }\end{cases} \\
& U_{2}{ }^{(l)}=\sum_{i=1}^{N_{\text {Area }}}\left|\sum_{j=1}^{N_{\mathrm{BS}}} \frac{N_{U_{\mathrm{BS}}}}{N_{\mathrm{S}}} \sum_{s=1}^{N_{\mathrm{S}}} \rho_{s, i, j}^{(l)}\left(\boldsymbol{W}^{(l)}\right)-\eta \mathcal{D}_{i} \mathcal{A}(i)\right| .
\end{aligned}
$$

The utility function expressed in (14) corresponds to the number of reference points covered by BSs. This utility is set to 0 if the cell capacity constraint expressed in (11) is not satisfied for at least one of the subareas. In other words, if the cell capacity constraint is not satisfied, we assume that particle $l$ does not cover the area at all. On the other hand, the second utility $U_{2}{ }^{(l)}$ computes the difference between the number of users served by particle $l$ and the minimum number of users that have to be served. In the case when all particles do not satisfy the cell capacity constraint (11), the PSO will aim to minimize $U_{2}$ until satisfying the cell capacity constraint. Once it finds a feasible solution, the PSO switches the utility to $U_{1}{ }^{(l)}$ and tries to minimize it until reaching $-\tau N_{\text {ref }}$.

At each iteration, PSO computes the global particle, denoted $\boldsymbol{W}^{\text {(global) }}$, that provides the best utility (i.e., either $U_{1}$ or $U_{2}$ depending on the feasibility of the particles in this iteration). In addition, for each particle $l$, PSO maintains a record of the position of its previous best performance, denoted $\boldsymbol{W}^{(1, \text { local })}$. Then, at each iteration $t$, PSO computes a velocity term $V_{w}^{(l)}, \forall w=1, \cdots, 2 N_{\mathrm{BS}}$ as follows:

$$
\begin{aligned}
V_{w}^{(l)}(t+1)= & \psi V_{w}^{(l)}(t)+c_{1} \phi_{1}\left(W_{w}^{(1, \text { local })}(t)-W_{w}^{(l)}(t)\right) \\
& +c_{2} \phi_{2}\left(W_{w}^{\text {global }}(t)-W_{w}^{(l)}(t)\right)
\end{aligned}
$$

where $\psi$ is the inertia weight and is used to control the convergence speed. It is usually chosen between 0.8 and 1.2. $c_{1}$ and $c_{2}$ represent the size of the step that the particle takes toward its best individual local candidate solution $W^{\text {(l,local) }}$ and the global best solution $W^{\text {global }}$, respectively. Usually, we choose $c_{1}=c_{2}$ and close to 2 . The parameters $\phi_{1}$ and $\phi_{2}$ are two random positive numbers generated for each $w$ (i.e., the 
element of the vector $\left.\boldsymbol{W}^{(l)}\right)$. Then, PSO updates each element $w$ of a particle $\boldsymbol{W}^{(l)}$ as follows:

$$
W_{w}^{(l)}(t+1)=W_{w}^{(l)}(t)+V_{w}^{(l)}(t+1) .
$$

This process is repeated until reaching convergence either by attaining the maximum number of iterations or by reaching the algorithm target (i.e., $U_{1} \leq-\tau N_{\text {ref }}$ ). Note that the target $U_{1}$ cannot be reached unless the cell capacity constraint is satisfied which is the case thanks to the introduction of $U_{2}$ in (15). Finally, after convergence, the PSO solution is given by $\boldsymbol{W}_{o p}=\boldsymbol{W}^{\text {global }}$. Details of the proposed algorithm are given in Algorithm 1. Although PSO's application has been proved to be effective, convergence to its most optimistic solution cannot be guaranteed in theory [26].

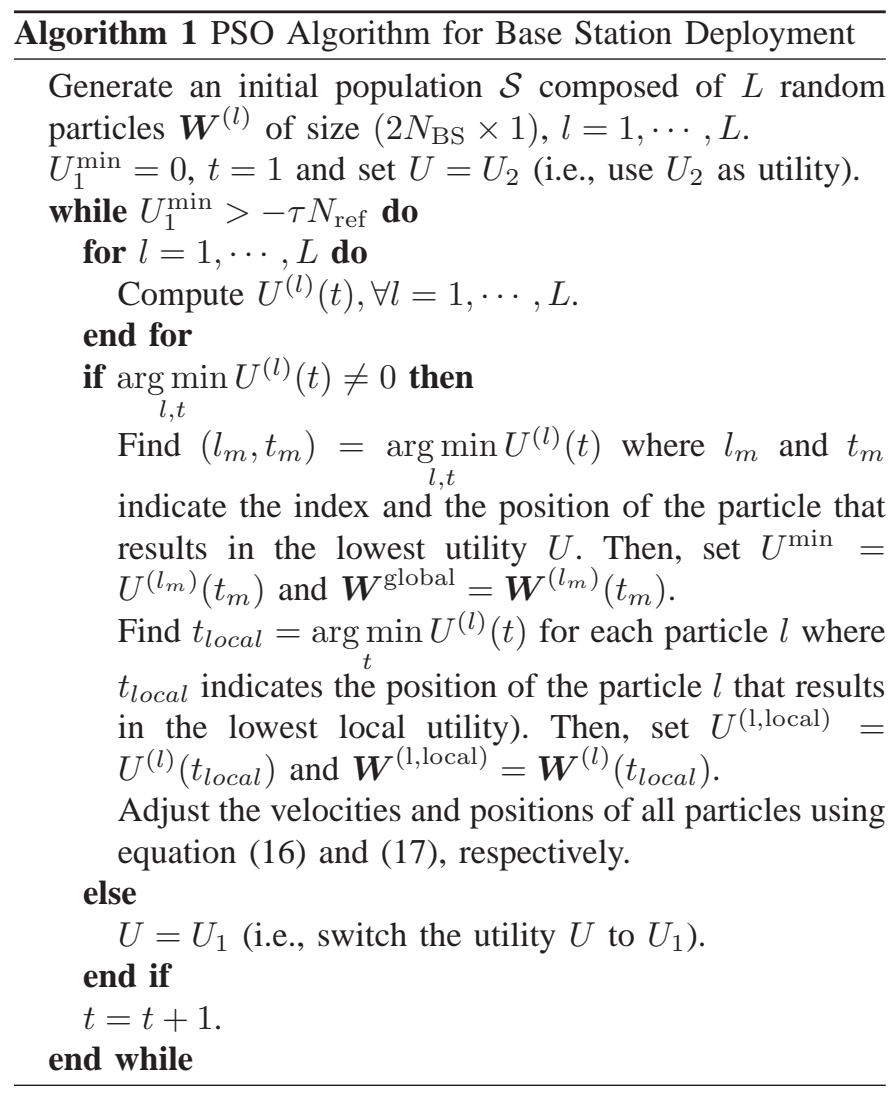

2) Grey Wolf Optimizer for Cell Planning: GWO is a new meta-heuristic algorithm proposed in [14]. It is inspired by grey wolves and it mimics the leadership hierarchy and hunting mechanism of grey wolves in nature. During an iteration, the algorithm categorizes the candidates (i.e., grey wolves) into four groups for simulating the leadership hierarchy: $\alpha$ corresponds to the fittest solution, $\beta$ and $\delta$ are the second and third best solutions. Finally, $\omega$ are the remaining candidates of the population. Also, the algorithm simulates the hunting, searching for prey, encircling prey, and attacking prey of grey wolves. The hunting corresponds to the position update of each candidate from an iteration to another (e.g., in our framework, it corresponds to the BS positions). It depends on the positions of the best candidates $\alpha, \beta$, and $\delta$ and is mathematically modeled as follows:

$$
\boldsymbol{W}^{(l)}(t+1)=\frac{1}{3}\left(\boldsymbol{W}_{\alpha 1}^{(l)}(t)+\boldsymbol{W}_{\beta 2}^{(l)}(t)+\boldsymbol{W}_{\delta 3}^{(l)}(t)\right),
$$

where $\boldsymbol{W}_{s i}^{(l)}(t)=\boldsymbol{W}_{s}^{(l)}(t)-\boldsymbol{A}_{\boldsymbol{i}} \cdot \boldsymbol{D}_{\boldsymbol{s}}$ for $(s, i) \in$ $\{(\alpha, 1),(\beta, 2),(\delta, 3)\}$ and $\boldsymbol{D}_{\boldsymbol{s}}=\left|\boldsymbol{C}_{\boldsymbol{i}} \cdot \boldsymbol{W}_{s}^{(l)}(t)-\boldsymbol{W}^{(l)}(t)\right|$. These equations model the encircling behavior of the prey. $A_{i}$ and $C_{i}$ are two coefficients vectors calculated as follows:

$$
A_{i}=2 a \cdot r_{1}-a, \text { and, } C_{i}=2 \cdot r_{2},
$$

where the components of the vector $\boldsymbol{a}$ are linearly decreased from 2 to 0 over the course of iterations and $r_{1}$ and $r_{2}$ are vectors randomly generated between $[0,1]$. The notation $(\boldsymbol{x} . \boldsymbol{y})$ corresponds to the vector dot product. $A_{\boldsymbol{i}}$ and $\boldsymbol{C}_{\boldsymbol{i}}$ influence the exploration for a better solution and are used to model the search for the prey while $\boldsymbol{a}$ is used to model the attacking behavior (i.e., approaching the prey) as it decreases over the course of iterations. To sum up, the search process in the GWO algorithm, starts with creating a random population of $L$ candidate solutions. Over the course of iterations, $\alpha, \beta$, and $\delta$ candidates estimate the probable position of the solution. Each candidate solution updates its distance using (18). The GWO algorithm is terminated by reaching the maximum number of iterations or satisfying the constraints (see [14]). Applied to our framework, we follow a procedure similar to the one used with PSO: we start by optimizing $U_{2}$ then $U_{1}$. Details of the proposed GWO algorithm are provided in Algorithm 2.

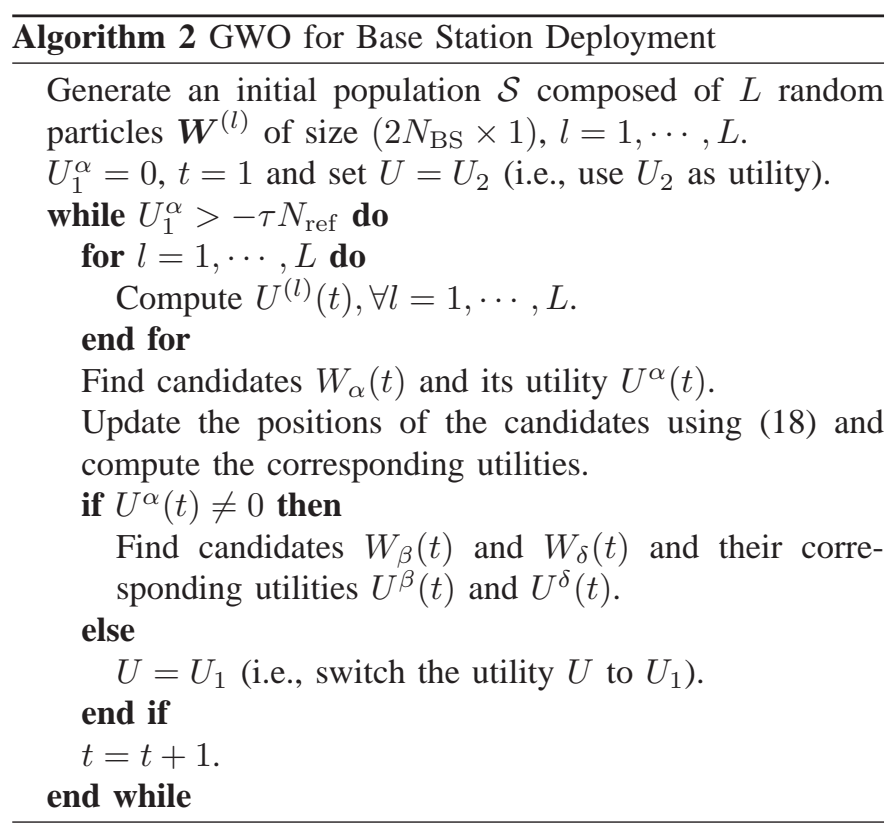

\section{B. Algorithm for Elimination of Redundant Base Stations}

In this step and after fixing the locations of the BSs using the meta-heuristic algorithm, we focus on the elimination of redundant BSs. A BS is considered useless if, when it is turned off, none of the cell capacity and coverage constraints is violated. In other words, if the absence of a BS affects at least one of the optimization problem constraints, the BS has to be kept and assumed indispensable for a safe network operation. In order to achieve this goal, we need to optimize the binary vector $\epsilon$ of size $\left(N_{\mathrm{BS}} \times 1\right)$ and find the optimal BS combination that does not affect the achieved meta-heuristic algorithm performance. Thus, we start by assuming that all BSs are placed in the area of interest (i.e., $\epsilon=[1, \cdots, 1]$ ). 
Then, we eliminate BS by BS and check, at each time, whether the problem constraints remain satisfied or not. If a BS $j$ degrades the problem performance, then it cannot be eliminated and $\epsilon_{j}$ remains 1 . Otherwise, the algorithm assumes that BS $j$ may be eliminated and places it in a set, denoted $\mathcal{E}$. After checking all BSs, the algorithm only needs to focus on the set $\mathcal{E}$ to identify the BSs that can be safely and completely eliminated and then set their corresponding $\epsilon_{j}$ to 0 .

It is not correct to eliminate all BSs in $\mathcal{E}$ simultaneously. Indeed, it may happen that two or more BSs in $\mathcal{E}$ support each other to maintain the coverage and/or cell capacity constraints. Thus, only one of them can be eliminated. We propose that this $\mathrm{BS}$, denoted $\hat{j}$, corresponds to the one that has the smallest impact on the number of served users as follows:

$$
\hat{j}=\underset{j \in \mathcal{E}}{\operatorname{argmax}} \sum_{i=1}^{N_{\text {Area }}} \sum_{j=1, j \neq \hat{j}}^{N_{\mathrm{BS}}}\left(\frac{N_{U_{\mathrm{BS}}}}{N_{\mathrm{S}}} \sum_{s=1}^{N_{\mathrm{S}}} \rho_{s, i, j}\left(\boldsymbol{W}_{o p}\right)-\eta \mathcal{D}_{i} \mathcal{A}(i)\right) .
$$

Note that we are actually sure that the term $\sum_{j=1}^{N_{\mathrm{BS}}} \frac{N_{U_{\mathrm{BS}}}}{N_{\mathrm{S}}} \sum_{s=1}^{N_{\mathrm{S}}} \rho_{s, i, j}\left(\boldsymbol{W}_{o p}\right)-\eta \mathcal{D}_{i} \mathcal{A}(i) \geq 0, \forall i=$ $1, \cdots, N_{\text {Area }}$ as we have already achieved a feasible solution with the planning algorithms proposed in Section III-A and we are maintaining in $\mathcal{E}$ only the BSs that do not violate the problem constraints. The procedure is repeated with the remaining BSs in $\mathcal{E}$ until obtaining the final BS combination. Details of the redundant BS elimination algorithm are given in Algorithm 3.

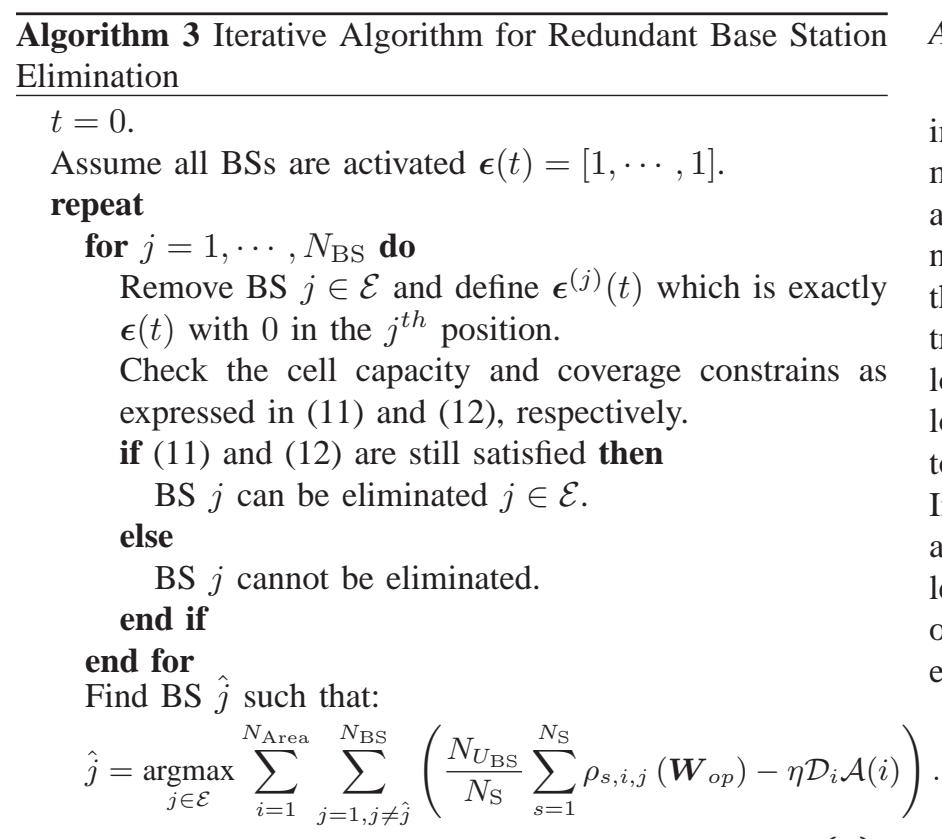

BS $\hat{j}$ is completely and safely eliminated, $\mathcal{E}=\mathcal{E} \backslash\{\hat{j}\}$, and $\boldsymbol{\epsilon}(t+1)=\boldsymbol{\epsilon}^{(\hat{j})}(t)$.$$
t=t+1 \text {. }
$$$$
\text { until } \mathcal{E}=\emptyset \text {. }
$$

The final BS combination after network planning is $\boldsymbol{\epsilon}(t)$.

Hence, the proposed planning approach consisted of two steps: A dimensioning phase where we determined the radius and number of BSs $\left(R_{\mathrm{BS}}, N_{\mathrm{BS}}\right)$ needed for the operation of the network and a BS deployment phase where we determined their suboptimal locations $(\boldsymbol{x}, \boldsymbol{y})$ in the geographical area after eliminating the eventual redundant BSs.

In practice, users can use different applications (e.g., voice, data, multimedia, etc) and can be divided into multiple classes with different expected data rates. Interestingly, the planning approach can be extended to support multiple services with different QoS requirements which are mainly treated in the dimensioning phase. First, we perform the coverage dimensioning phase by computing the MAPL in uplink and downlink after establishing the radio link budget related to each service $i$. Next, we identify the minimum MAPL $\left(\mathrm{MAPL}_{\min }=\right.$ $\left.\operatorname{argmin}_{i} \mathrm{MAPL}_{i}\right)$ and determine the corresponding base station radius and thus the estimated number of base stations that are needed to cover the area. Concerning the capacity dimensioning, we can compute the average target data rate threshold in (2) as a weighted sum of the data rate threshold of each service $\left(\sum_{i} \alpha_{i} R_{\mathrm{th}, i}^{(\mathrm{DL})}\right)$ where $\alpha_{i}$ reflects the percentage of subscribers in the network using service $i$. Finally, we apply our planning approach in order to place the base stations. Next, in the Monte Carlo simulation, we place users following a given distribution such that each user is using one of the services following the occurrence probability of the service defined by $\alpha_{i}$. The outage rate is determined by comparing the achieved data rate of each user with the corresponding service data rate threshold.

\section{Applications: Green Planning And LocAtion CONSTRAINTS}

\section{A. Green Planning with Temporal Traffic Considerations}

In this section, we propose a planning method that takes into account the temporal traffic variation. This method allows mobile operators to forecast the switching on/off of BSs according to known traffic behaviour changes, without the need to assess the problem continuously. It consists of finding the number of BSs per subarea needed to serve users in low traffic periods (e.g., the night period) in addition to their locations to ensure the coverage. Then, it determines the locations of the additional BSs to be placed in the area in order to fit higher traffic period constraints (e.g., the day period). In the sequel, we will consider the Day/Night case but the approach can be extended to a larger number of traffic density levels (e.g., high, medium, low) that could happen any time of the day, not just two levels (day, night). The algorithm is executed as follows

- Step 1: The coverage and the cell capacity dimensioning phase is performed to find the required number of BSs $N_{\mathrm{BS}}^{\text {Night }}$ needed for the night period.

- Step 2: The proposed method described in Section III is employed in order to solve the optimization formulation in (26) for the night traffic by optimization the locations of the $N_{\mathrm{BS}}^{\text {Night }}$ BSs denoted $\left(\boldsymbol{x}_{\boldsymbol{N}}, \boldsymbol{y}_{\boldsymbol{N}}\right)$.

- Step 3: The coverage and the cell capacity dimensioning phase is again performed to find the required number of BSs $N_{\mathrm{BS}}^{\mathrm{Day}}$ needed for the day period.

- Step 4: The proposed method described in Section III is employed in order to solve the following constrained optimization problem for the day traffic by only optimizing the locations of the new added BSs (i.e., $N_{\mathrm{BS}}^{\mathrm{Day}}-N_{\mathrm{BS}}^{\mathrm{Night}}$ 
BSs) having as coordinates $(\boldsymbol{x}, \boldsymbol{y})$ and considering that $N_{\mathrm{BS}}^{\text {Night }}$ BSs obtained in Step 2 are already deployed:

$$
\underset{\boldsymbol{x}, \boldsymbol{y}, \boldsymbol{\epsilon}}{\operatorname{Minimize}} \sum_{j=1}^{N_{\mathrm{BS}}^{\text {Day }}} \epsilon_{j}
$$

Subject to:

$$
\begin{aligned}
& \sum_{j=1}^{N_{\mathrm{BS}}^{\text {Day }}} \sum_{s=1}^{N_{\mathrm{S}}} \frac{N_{U_{\mathrm{BS}}}}{N_{\mathrm{S}}} \rho_{s, i, j}\left(\left[\boldsymbol{x}, \boldsymbol{x}_{\boldsymbol{N}}\right]^{T},\left[\boldsymbol{y}, \boldsymbol{y}_{\boldsymbol{N}}\right]^{T}\right) \geq \eta \mathcal{D}_{i} \mathcal{A}(i), \\
& \forall i=1, \cdots, N_{\text {Area }} \\
& \sum_{n=1}^{N_{\text {ref }}} \gamma_{n}\left(\left[\boldsymbol{x}, \boldsymbol{x}_{\boldsymbol{N}}\right]^{T},\left[\boldsymbol{y}, \boldsymbol{y}_{\boldsymbol{N}}\right]^{T}\right) \geq \tau N_{\text {ref }}
\end{aligned}
$$

This green planning approach helps in ensuring energy saving by identifying the BSs that will be always active on day and night (referred to as the "night BSs") and the BSs that need to be activated only during the day (referred to as the "day BSs") to handle the increased traffic load, but can be switched off at low traffic periods at night with respect to the user densities in the subareas.

\section{B. Planning Subject to Location Constraints}

During the planning phase, mobile operators have to respect some location constraints. Positions that are found through the proposed approach may not be available for installing BSs in real life due to several reasons. For example, the location may fall in a private property, or restricted access area, or radio-sensitive zones such as schools or hospitals. Thus, these location constraints should be taken into account during the planning phase. In this section, we exploit the efficiency of the proposed method in order to perform the BS planning while respecting this constraint which is expressed as follows

$$
\left(x_{j}, y_{j}\right) \notin \mathcal{S}_{R}, \quad \forall j=1, \cdots, N_{\mathrm{BS}},
$$

where $\mathcal{S}_{R}$ denotes the restricted areas in the entire region where BSs cannot be placed. In the context of radiationsensitive zones, the constraint can be defined such that the total received power at each reference point $n$ in this zone has to be below a certain threshold. The total received power $\mathrm{PR}_{n}$ corresponds to the sum of power received from all BSs while the power threshold $P_{\mathrm{th}}$ can be determined from [27]. Health recommendations suggest that the median exposure in urban areas be limited to $0.005 \mu \mathrm{W} / \mathrm{cm}^{2}$ and that $95 \%$ of the urban population be exposed to less than $0.1 \mu \mathrm{W} / \mathrm{cm}^{2}$. Therefore, the electromagnetic radiation constraint at each reference point $n$ can be written as follows:

$$
\mathrm{PR}_{n}=\sum_{j=1}^{N_{\mathrm{BS}}} \sum_{s=1}^{N_{\mathrm{S}}} \frac{\mathrm{P}_{j, s}}{\mathrm{PL}_{\mathrm{j}, \mathrm{s}, \mathrm{n}}} \leq P_{\mathrm{th}}, \quad \forall n \in \mathcal{S}_{R}
$$

where $\mathrm{P}_{j, s}$ is the BS $j$ transmit power emitted by sector $s$ while $\mathrm{PL}_{\mathrm{j}, \mathrm{s}, \mathrm{n}}$ is the pathloss between sector $s$ of BS $j$ and reference point $n$. Thus, the optimization problem to be solved for the radiation-sensitive zone problem can be written as follows:

$$
\underset{\boldsymbol{x}, \boldsymbol{y}, \boldsymbol{\epsilon}}{\operatorname{Minimize}} \sum_{j=1}^{N_{\mathrm{BS}}} \epsilon_{j},
$$

Subject to:

$\sum_{j=1}^{N_{\mathrm{BS}}} \sum_{s=1}^{N_{\mathrm{S}}} \frac{N_{U_{\mathrm{BS}}}}{N_{\mathrm{S}}} \rho_{s, i, j}(\boldsymbol{x}, \boldsymbol{y}) \geq \eta\left(\mathcal{S}_{R}\right) \mathcal{D}_{i} \mathcal{A}(i), \forall i=1, \cdots, N_{\text {Area }}$,

$\sum_{n=1}^{N_{\text {ref }}} \gamma_{n}(\boldsymbol{x}, \boldsymbol{y}) \geq \tau\left(\mathcal{S}_{R}\right) N_{\text {ref }}$

$\sum_{j=1}^{N_{\mathrm{BS}}} \sum_{s=1}^{N_{\mathrm{S}}} \frac{\mathrm{P}_{j, s}}{\mathrm{PL}_{\mathrm{j}, \mathrm{s}, \mathrm{n}}} \leq P_{\mathrm{th}}, \quad \forall n \in \mathcal{S}_{R}$

Note that, in this problem, the coverage and capacity tolerances (i.e., $\tau$ and $\eta$ ) are now in function of the radiation free zone $\mathcal{S}_{R}$ and must be adapted to the new location constraint as in some cases, the constraints can be contradictory. For instance, if the radiation-sensitive zone is relatively large comparing to the cell range, then some reference points will not be covered. Thus, a particular choice of $\tau\left(\mathcal{S}_{R}\right)$ and $\eta\left(\mathcal{S}_{R}\right)$ could be $\tau\left(\mathcal{S}_{R}\right)=\left(1-\left|\mathcal{S}_{R}\right|\right)$ and $\eta\left(\mathcal{S}_{R}\right)=1 / \mathcal{S}_{R}$, respectively where $\left|\mathcal{S}_{R}\right|$ denotes the cardinality of the set $\mathcal{S}_{R}$ and $\mathcal{A}(i) / \mathcal{S}_{R}$ represents the set subtraction and corresponds to the area of $\mathcal{A}(i)$ that does not intersect with $\mathcal{S}_{R}$.

The problem could be solved using the same method described in Section III but by considering the new constraint. This constraint can be converted to a utility function as it is done in (14) and (15). The radiation-sensitive zone utility achieved by particle $l, U_{3}{ }^{(l)}$, can be then expressed as

$$
U_{3}{ }^{(l)}=\left|\mathcal{S}_{R}\right|-N_{P},
$$

where $N_{P}$ denotes the number of reference points that satisfies the constraint given in (25). Thus, the algorithm can be executed to first minimize $U_{3}$ until reaching zero, then it switches the utility in order to optimize the cell capacity and coverage constraints.

\section{Performance Evaluation Method}

After deploying the LTE network BSs in their appropriate locations according to Section III, we apply a Monte Carlo simulation in order to investigate the impact of the proposed cell planning approach on the uplink and downlink scheduling while taking intercell interference into account, thus measuring the efficiency of the proposed scheme in realistic scenarios similar to those adopted in the literature, e.g., [28]-[30]. In each realization, we distribute $N_{\mathrm{U}}$ users following the distributions defined for each subarea $i$ of the geographical area. Then, we verify whether a user $u$ is served successfully or not by comparing their achieved data rates, denoted $R_{u}{ }^{(\mathrm{DL})}$ and $R_{u}{ }^{(\mathrm{UL})}$, to the target data rate thresholds, denoted $R_{\mathrm{th}}{ }^{(\mathrm{DL})}$ and $R_{\mathrm{th}}{ }^{(\mathrm{UL})}$, for the DL and UL directions, respectively. The objective is to determine the average outage rate which has to be in harmony with the imposed tolerance during the planning phase. In order to compute the data rates, we need to define the channel gain over RB $r$ between user $u$ and sector $s$ of 
BS $j$ as follows:

$H_{u, r, s, j, \mathrm{~dB}}=\left(-\kappa-v \log _{10} d_{u, j}\right)-\xi_{u, r, s, j}+10 \log _{10} F_{u, r, s, j}$.

In (31), the first term captures the propagation loss, with $\kappa$ the pathloss constant, $d_{u, j}$ the distance in $\mathrm{km}$ from user $u$ to BS $j$, and $v$ the path loss exponent. The second term, $\xi_{u, r, s, j}$, captures log-normal shadowing with zero-mean and a standard deviation $\sigma_{\xi}$, whereas the last term, $F_{u, r, s, j}$, corresponds to the Rayleigh fading with a Rayleigh parameter $\bar{a}$ (usually selected such that $\mathbb{E}\left[\bar{a}^{2}\right]=1$ ). Note that in the radio link budget presented in Table I, we compensate for shadowing effects using two power margins (slow fading margin and shadowing handover gain); the values of the power margins are determined based on the shadowing statistics. This is the common approach used by cellular operators in their link budget analysis to capture statistical variations. However, the channel model in the Monte-Carlo simulations includes both shadowing and fast fading to verify that the planning method's outcome is able indeed to meet the target performance requirements.In the sequel, in order to differentiate between UL and DL RBs, the notation $H_{u, r, s, j}^{(\mathrm{UL})}$ and $H_{u, r, s, j}^{(\mathrm{DL})}$ will be used, respectively.

\section{A. Downlink and Uplink Data Rates}

Letting $\mathcal{I}_{\mathrm{RB}, u}^{(\mathrm{DL})}$ the set of RBs allocated to user $u$ in the DL, $N_{\mathrm{RB}}^{(\mathrm{DL})}$ the total number of DL RBs, $P_{r}$ the power transmitted by a BS over RB $r$, and $P_{\max }$ the maximum transmission power of BS $j$. Then, the OFDMA throughput of user $u$ in the DL direction is given by:

$$
R_{u}^{(\mathrm{DL})}\left(P_{j, \max }^{\mathrm{tx}}, \mathcal{I}_{\mathrm{RB}, u}^{(\mathrm{DL})}\right)=\sum_{r \in \mathcal{I}_{\mathrm{RB}, u}^{(\mathrm{DL})}} B_{\mathrm{RB}}^{(\mathrm{DL})} \cdot \log _{2}\left(1+\Gamma_{u, r, s, j}^{(\mathrm{DL})}\right),
$$

where $\Gamma_{u, r, s, j}^{(\mathrm{DL})}$ is the SINR of user $u$ over RB $r$ in cell sector $s$ of $\mathrm{BS} j$, and $B_{\mathrm{RB}}^{(\mathrm{DL})}$ is the $\mathrm{RB}$ bandwidth. It is expressed as:

$$
B_{\mathrm{RB}}^{(\mathrm{DL})}=\frac{B^{(\mathrm{DL})}}{N_{\mathrm{RB}}^{(\mathrm{DL})}},
$$

with $B^{(\mathrm{DL})}$ the total usable DL bandwidth, and $N_{\mathrm{RB}}^{(\mathrm{DL})}$ the total number of DL RBs. In this paper, we consider equal power transmission over the RBs, i.e., for all $r$, we have:

$$
P_{r}=\frac{P_{\max }}{N_{\mathrm{RB}}^{(\mathrm{DL})}} .
$$

The DL-SINR of user $u$ over $\mathrm{RB} r$ in $\mathrm{BS} j, \Gamma_{u, r, s, j}^{(\mathrm{DL})}$, is given by:

$$
\Gamma_{u, r, s, j}^{(\mathrm{DL})}=\frac{P_{r} G_{u, s, j}^{\mathrm{BS}} G^{\mathrm{MS}} H_{u, r, s, j}^{(\mathrm{DL})}}{I_{r, u}^{(\mathrm{DL})}+\sigma_{r, u}^{2}},
$$

where $\sigma_{r, u}^{2}$ is the noise power over $\mathrm{RB} r$ in the receiver of user $u$ and is considered constant and equal to $\left(K T B_{\mathrm{RB}}\right)$ where $K$ is the Boltzmann constant and $T$ is the ambient temperature (300 Kelvin), $G^{\mathrm{MS}}$ is the omnidirectional antenna gain of the MS, and $G_{u, s, j}^{\mathrm{BS}}$ is the BS antenna gain which is modeled as proposed in [31] and with simplification introduced in [32] as

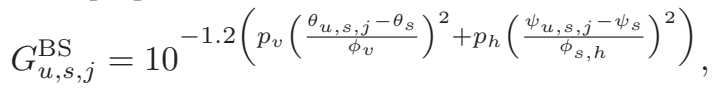

where $\theta_{u, s, j}=\arctan \left(\frac{h_{\mathrm{BS}}}{d_{u, j}}\right)$ is the vertical angle in degrees from sector $s$ of $\mathrm{BS} j$ to user $u$. The $\psi_{u, s, j}$ is horizontal angle in degrees on sector $s$ of BS $j$ and user $u$ with respect to positive $\mathrm{x}$-axis. Subscripts $h$ and $v$ denote horizontal and vertical respectively. Thus, $p_{h}$ and $p_{v}$ represent weighting factors for the horizontal and vertical beam pattern of the antenna in 3D antenna model [31], respectively. Note that for the practical cellular antennas the relationship between the horizontal beamwidth of sector antenna and the number of sectors per site can modeled as $\phi_{s, h}=\frac{360}{\mu N_{\mathrm{S}}}$ where $\mu$ is a factor representing overlap between the sectors. Finally, $\theta_{s}$ and $\phi_{s}$ represent the tilt and the azimuth angles of sector $s$, respetively, while $\phi_{v}$ denotes the vertical beamwidth of the antenna. In (35), $I_{r, u}^{(\mathrm{DL})}$ is the interference on RB $r$ measured at the receiver of user $u$. The expression of the interference is given by:

$$
I_{r, u}=\sum_{k=1, k \neq j}^{N_{\mathrm{BS}}}\left(\sum_{v=1}^{N_{\mathrm{U}}} \lambda_{v, r, k}^{(\mathrm{DL})}\right) P_{r} G_{u, s, k}^{\mathrm{BS}} G^{\mathrm{MS}} H_{u, r, s, k}^{(\mathrm{DL})},
$$

where $\lambda_{v, r, k}^{(\mathrm{DL})}=1$ if DL RB $r$ is allocated to user $v$ in cell $k$, i.e., $r \in \mathcal{I}_{\mathrm{RB}, v}^{(\mathrm{DL})}$. Otherwise, $\lambda_{v, r, k}=0$. In each cell, an LTE $\mathrm{RB}$, and hence the subcarriers constituting that RB, can be allocated to a single user at a given time transmission interval. Hence, in each cell $k$, we have:

$$
\sum_{v=1}^{N_{\mathrm{U}}} \lambda_{v, r, k}^{(\mathrm{DL})} \leq 1
$$

Concerning the UL direction, assume that $\mathcal{I}_{\mathrm{RB}, u}^{(\mathrm{UL})}$ the set of UL RBs allocated to user $u, N_{\mathrm{RB}}^{(\mathrm{UL})}$ the total number of RBs in the UL, and $P_{u}^{(\mathrm{MS})}$ the total transmit power of user $u$. Then, the SC-FDMA throughput of user $u$ in the UL direction is given by:

$$
\begin{aligned}
& R_{u}^{(\mathrm{UL})}\left(P_{u}^{(\mathrm{MS})}, \mathcal{I}_{\mathrm{RB}, u}^{(\mathrm{UL})}\right)= \\
& \quad \frac{B^{(\mathrm{UL})}\left|\mathcal{I}_{\mathrm{RB}, u}^{(\mathrm{UL})}\right|}{N_{\mathrm{RB}}^{(\mathrm{UL})}} \log _{2}\left(1+\Gamma_{u}^{(\mathrm{UL})}\left(P_{u}^{(\mathrm{MS})}, \mathcal{I}_{\mathrm{RB}, u}^{(\mathrm{UL})}\right)\right),
\end{aligned}
$$

where $B^{(\mathrm{UL})}$ is the total UL bandwidth, $\left|\mathcal{I}_{\mathrm{RB}, u}^{(\mathrm{UL})}\right|$ is the cardinality of $\mathcal{I}_{\mathrm{RB}, u}^{(\mathrm{UL})}$ and $N_{\mathrm{RB}}^{(\mathrm{UL})}$ is the number of UL RBs. Finally, $\Gamma_{u}^{(\mathrm{UL})}\left(P_{u}^{(\mathrm{MS})}, \mathcal{I}_{\mathrm{RB}, u}^{(\mathrm{UL})}\right)$ is the SINR of user $u$ after Minimum Mean Squared Error (MMSE) frequency domain equalization at the receiver [33]:

$$
\Gamma_{u}^{(\mathrm{UL})}\left(P_{u}^{(\mathrm{MS})}, \mathcal{I}_{\mathrm{RB}, u}^{(\mathrm{UL})}\right)=\left(\frac{1}{\frac{1}{\left|\mathcal{I}_{\mathrm{RB}, u}^{(\mathrm{UL})}\right|} \sum_{r \in \mathcal{I}_{\mathrm{RB}, u}^{(\mathrm{UL})}} \frac{\Gamma_{u, r, s, j}^{(\mathrm{UL})}}{\Gamma_{u, r, s, j}^{(\mathrm{UL})}+1}}-1\right)^{-1}
$$

In (40), $\Gamma_{u, r, s, j}^{(\mathrm{UL})}$ is the UL SINR of user $u$ over RB $r$ served by sector $s$ of $\mathrm{BS} j$. It is given by:

$$
\Gamma_{u, r, s, j}^{(\mathrm{UL})}=\frac{P_{u, r, j}^{(\mathrm{UL})} G_{u, s, j}^{\mathrm{BS}} G^{\mathrm{MS}} H_{u, r, s, j}^{(\mathrm{UL})}}{I_{r, j}^{(\mathrm{UL})}+\sigma_{r, j}^{2}},
$$

where $H_{u, r, s, j}^{(\mathrm{UL})}$ is the channel gain between user $u$ and BS $j$ over RB $r, \sigma_{r, j}^{2}$ is the noise power over subcarrier $r$ at BS $j$, $P_{u, r, j}^{(\mathrm{UL})}$ is the power transmitted by user $u$ over subcarrier $r$ in 
BS $j$, and $I_{r, j}^{(\mathrm{UL})}$ is the UL interference on RB $r$, measured at BS $j$. The expression of the interference is given by:

$$
I_{r, j}^{(\mathrm{UL})}=\sum_{k=1, k \neq j}^{N_{\mathrm{BS}}} \sum_{v=1}^{N_{\mathrm{U}}} \lambda_{v, r, k}^{(\mathrm{UL})} P_{v, r, k}^{(\mathrm{UL})} G_{v, s, j}^{\mathrm{BS}} G^{\mathrm{MS}} H_{v, r, s, j}^{(\mathrm{UL})}
$$

where $\lambda_{v, r, k}^{(\mathrm{UL})}=1$ if RB $r$ is allocated to user $v$ served by BS $k$, i.e., $r \in \mathcal{I}_{\mathrm{RB}, v}^{(\mathrm{UL})}$. Otherwise, $\lambda_{v, r, k}^{(\mathrm{UL})}=0$. The LTE standard imposes the constraint that the RBs allocated to a single user should be consecutive with equal power allocation over the RBs [34], [35]. Hence, we set:

$$
P_{u, r, j}^{(\mathrm{UL})}=\frac{P_{u}^{(\mathrm{MS})}}{\left|\mathcal{I}_{\mathrm{RB}, u}^{(\mathrm{UL})}\right|} .
$$

\section{B. Admission Control and Resource Allocation}

We assume that the subcarriers constituting a single RB are subjected to the same fading and hence the channel gain on the subcarriers of a single RB is considered to be the same. In addition, the fading is assumed to be independent identically distributed (iid) across RBs [36]. In accordance with the radio link budgets given in Table I (i.e., computation of the thermal noise), we allocate one UL RB and one DL RB for each user. Note that the proposed method can indeed be applied with any scheduling algorithm.

Hence, when a user $u$ joins the network, it is associated with sector $s^{*}$ of cell $j^{*}$ and the DL RB for which the RB $r^{*(\mathrm{DL})}$ satisfy:

$$
\left(r^{*(\mathrm{DL})}, s^{*}, j^{*}\right)=\arg \max _{(r, s, j)}\left(1-\sum_{v=1 ; v \neq u}^{U} \lambda_{v, r, j}^{(\mathrm{DL})}\right) H_{u, r, s, j}^{(\mathrm{DL})} .
$$

Then, for the UL, it is allocated the RB in sector $s^{*}$ of cell $j^{*}$ for which the RB $r^{*(\mathrm{UL})}$ satisfies:

$$
r^{*(\mathrm{UL})}=\arg \max _{r}\left(1-\sum_{v=1 ; v \neq u}^{U} \lambda_{v, r, j^{*}}^{(\mathrm{UL})}\right) H_{u, r, s^{*}, j^{*}}^{(\mathrm{UL}}
$$

In (44) and (45), the first factor in the multiplication indicates that the search is on the RBs that are not yet allocated to other users. Then, the rates (32) and (39) are computed. We start by allocating DL subcarriers first in order to save BS power usage since usually the DL traffic is much heavier than UL traffic.

Finally, a user $u$ is considered to be successfully served if the following conditions are satisfied:

$$
\left\{\begin{array}{l}
R_{u}^{(\mathrm{UL})} \geq R_{\mathrm{th}}^{(\mathrm{UL})} \\
R_{u}^{(\mathrm{DL})} \geq R_{\mathrm{th}}^{(\mathrm{DL})}
\end{array}\right.
$$

\section{RESUlTS AND DiscusSiON}

In this section, after detailing the system parameters, we analyze the performance of the BS deployment method presented in Section III. Then, we study the performance of the proposed scheme considering the existence of femtocells, using the green planning and the radiation-sensitive zone problems.

\section{A. Simulation Model}

\begin{tabular}{|c|c|}
\hline Data rate (Mbps) & 1 \\
\hline \multicolumn{2}{|l|}{ Transmitter - BS } \\
\hline a $\quad$ BS power $(\mathrm{dBm})$ & 46 \\
\hline b TX antenna gain $(\mathrm{dBi})$ & 18 \\
\hline c Cable loss $(\mathrm{dB})$ & 2.0 \\
\hline d $\operatorname{EIRP}(\mathrm{dBm})$ & $62.0=a+b-c$ \\
\hline \multicolumn{2}{|l|}{ Receiver-MS } \\
\hline e $\quad$ MS noise figure $(\mathrm{dB})$ & 7.0 \\
\hline f Thermal noise $(\mathrm{dBm})$ & $-121.27=10 \log _{10}\left(K T B_{R B}\right)+30$ \\
\hline g Receiver noise floor $(\mathrm{dBm})$ & $-114.27=e+f$ \\
\hline h $\operatorname{SINR}(\mathrm{dB})$ & -10.0 \\
\hline Receiver sensitivity (dBm) & $-124.27=g+h$ \\
\hline Interference Margin $(\mathrm{dB})$ & 2.0 \\
\hline $\mathrm{RX}$ antenna gain $(\mathrm{dBi})$ & 0.0 \\
\hline 1 Body Loss (dB) & 0.0 \\
\hline \multicolumn{2}{|c|}{$\begin{array}{l}\text { DL MAPL: } 159.77=d-(i+j+k-l+S F M-S H G+P e L) \\
\text { where } S F M: \text { Slow Fading Margin }(=9 d B), \\
\text { SHG: Shadowing Handover gain }(=2.5 d B), \\
\text { PeL: Penetration Loss }(=18 d B) .\end{array}$} \\
\hline
\end{tabular}

We consider a $10 \times 10\left(\mathrm{~km}^{2}\right)$ LTE coverage area where we aim to deploy a certain number of three sector antenna BSs

\begin{tabular}{|c|c|}
\hline Data rate (Kbps) & 64 \\
\hline \multicolumn{2}{|l|}{ Transmitter - MS } \\
\hline a $\quad$ MS power $(\mathrm{dBm})$ & 23 \\
\hline $\mathrm{TX}$ antenna gain $(\mathrm{dBi})$ & 0 \\
\hline Cable loss (dB) & 0 \\
\hline d $\operatorname{EIRP}(\mathrm{dBm})$ & $23.0=a+b-c$ \\
\hline \multicolumn{2}{|l|}{ Receiver - BS } \\
\hline e $\quad$ BS noise figure $(\mathrm{dB})$ & 2.5 \\
\hline f Thermal noise $(\mathrm{dBm})$ & $-121.27=10 \log _{10}\left(K T B_{R B}\right)+30$ \\
\hline g Receiver noise floor (dBm) & $-118.77=e+f$ \\
\hline $\operatorname{SINR}(\mathrm{dB})$ & -7.0 \\
\hline Receiver sensitivity (dBm) & $-125.77=g+h$ \\
\hline Interference Margin (dB) & 1.0 \\
\hline Cable Loss $(\mathrm{dB})$ & 2.0 \\
\hline $\mathrm{RX}$ antenna gain $(\mathrm{dBi})$ & 18 \\
\hline m MHA gain $(\mathrm{dB})$ & 2.0 \\
\hline \multicolumn{2}{|c|}{$\begin{array}{l}\text { UL MAPL: } 137.27=d-(i+j+k-l+m+S F M-S H G+P e L) \\
\text { where } S F M \text { : Slow Fading Margin }(=9 d B), \\
\text { SHG: Shadowing Handover gain }(=2.5 d B), \\
\text { PeL: Penetration Loss }(=18 d B) .\end{array}$} \\
\hline
\end{tabular}

Table I: Radio link budgets

Downlink Link Budget for $1 \mathrm{Mbps}$

(a)

(b)

TABLE II: Channel and power parameters

\begin{tabular}{|c|c||c|c|}
\hline Parameter & Value & Parameter & Value \\
\hline \hline$\kappa(\mathrm{dB})$ & -128.9 & $v$ & 34.4 \\
\hline$\sigma_{\xi}(\mathrm{dB})$ & 8 & $N_{\mathrm{RB}}^{(\mathrm{x})}$ & 50 \\
\hline$B^{(\mathrm{x})}(\mathrm{MHz})$ & 10 & $B_{\text {sub }}^{(\mathrm{x})}(\mathrm{kHz})$ & 15 \\
\hline $\mathrm{BS}$ power $(\mathrm{dBm})$ & 46 & MS power $(\mathrm{dBm})$ & 23 \\
\hline
\end{tabular}

$\left(N_{\mathrm{S}}=3\right)$ in order to cover the whole area while respecting the cell capacity constraints in the given subareas. The initial number of BSs to be deployed $N_{\mathrm{BS}}$, the cell range $R_{\mathrm{BS}}$, and the number of users that can be served by a BS simultaneously $N_{U_{\mathrm{BS}}}$ are computed after elaborating the dimensioning phase as described in Section II-A using the DL and UL budgets given in Table I(a) and Table I(b), respectively, and the COST231-HATA propagation model [19]. From these tables and for a frequency carrier equal to $1.8 \mathrm{GHz}, h_{\mathrm{BS}}=40 \mathrm{~m}$, and $h_{\mathrm{MS}}=1.5 \mathrm{~m}$, we find that $R_{\mathrm{BS}}=1.2 \mathrm{~km}$. We assume that the target data rate in UL direction is $64 \mathrm{kbps}$ while, in the DL, the required data rate is $1 \mathrm{Mbps}$ for each user. In addition, we assume that the system bandwidth is equal to 
TABLE III Studied Scenarios And the CorResponding Performance

\begin{tabular}{|c||c|c|c||c|c|c|c|c|}
\hline Scenarios & $\boldsymbol{N}_{\mathrm{U}}$ & $\boldsymbol{N}_{\text {Area }}$ & Subarea Description & $\boldsymbol{N}_{\mathrm{BS}}$ & $\sum_{j=1}^{N_{\mathrm{BS}}} \boldsymbol{\epsilon}_{\boldsymbol{j}}$ & \multicolumn{2}{|c|}{ Outage rate } \\
\hline \hline & & & & & PSO & GWO & PSO & GWO \\
\hline Scenario A & 2000 & 2 & $\begin{array}{c}\text { Subarea } 1 \text { is uniformly distributed, } \\
\text { Subarea } 2 \text { is normally distributed } \\
\text { reaching its maximum at }(5 \mathrm{~km}, 5 \mathrm{~km}) \\
\left(A(1), D_{1}\right)=\left(65 \mathrm{~km}^{2}, 40 \%\right) \\
\left(A(2), D_{2}\right)=\left(35 \mathrm{~km}^{2}, 60 \%\right)\end{array}$ & 42 & 41 & 41 & $0.5 \%$ & $0.62 \%$ \\
Scenario B & 2000 & 2 & $\begin{array}{c}\text { Subarea } 1 \text { is uniformly distributed, } \\
\text { Subarea } 2 \text { is uniformly distributed } \\
\left(A(1), D_{1}\right)=\left(65 \mathrm{~km}^{2}, 40 \%\right) \\
\left(A(2), D_{2}\right)=\left(35 \mathrm{~km}^{2}, 60 \%\right)\end{array}$ & 42 & 40 & 40 & $0.3 \%$ & $0.36 \%$ \\
Scenario C & 1000 & 4 & $\begin{array}{c}\text { All subareas are uniformly distributed } \\
(A(1), D(1))=\left(33.3 \mathrm{~km}^{2}, 35 \%\right) \\
(A(2), D(2))=\left(16.6 \mathrm{~km}^{2}, 40 \%\right) \\
(A(3), D(3))=\left(16.6 \mathrm{~km}^{2}, 5 \%\right) \\
(A(4), D(4))=\left(33.3 \mathrm{~km}^{2}, 20 \%\right)\end{array}$ & 33 & 33 & 33 & $0.21 \%$ & $0.26 \%$ \\
\hline
\end{tabular}

$B=10 \mathrm{MHz}$ while the DL spectral efficiency is fixed to be 1.74 (bit/s/Hz/cell) using $2 \times 2$ MIMO [16]. Thus, $N_{U_{\mathrm{BS}}}=51$ users per 3-sector BS.

The GWO and PSO algorithms are applied under the following settings: the initial population size is set to $L=12$ while the tolerances $\eta$ and $\tau$ are set to $\eta=\tau=98 \%$. Also for PSO, we define $V_{\max }$ as the maximum achieved velocity in (16) (i.e., $\left.V_{w}^{(l)} \in\left[-V_{\max }, V_{\max }\right]\right)$. Indeed, this restriction is placed to enforce the limitation that a particle does not exceed a certain acceleration. We choose $V_{\max }=500$ meters in order to limit the movement of BSs from an iteration to another. The power and channel parameters are detailed in Table II and are obtained from [37], [38].

\section{B. Performance of the Proposed Planning Approach}

In our simulation results, we start by investigating the performance of the proposed approach using the PSO algorithm. We consider two scenarios, Scenario A and B as describes Table III, where in Scenario A we assume that the users in subarea 1 representing $60 \%$ of the total number of users are normally distributed with a concentration in the center of the area. Then, the number of users is reduced with a standard deviation of 1275 meter. However, in Scenario B, the users are uniformly distributed in subarea 1. In Fig. 2, we compare between the results obtained for both scenarios: Scenario A (i.e., Fig. 2(a)) and Scenario B (i.e., Fig. 2(b)). Although we are considering the same subareas and the same number of users, results show that the number of BSs required to fulfill the problem constraints is 41 for Scenario A and 40 for Scenario B with one extra BS for Scenario A. We also notice that Scenario A presents a higher BS density in subarea 1 compared to Scenario B. Indeed, the algorithm adapts its BS distribution to the user density without affecting the coverage constraint. Also, the outage rate for both scenarios is low around $0.5 \%$ and $0.3 \%$ for Scenario A and Scenario B, respectively.

The BSs in the boundary play an important role as they contribute in serving users in multiple subareas. We notice that, for the Gaussian scenario, the boundary BSs placed in subarea 2 contribute more in serving users placed in subarea 1 than the uniform scenario, since the user density in the boundary is very low for Scenario A.

\section{Comparison between PSO and GWO}

In Scenario C, the area of interest is divided into 4 subareas and users are uniformly distributed according to different densities as indicates Table III. In order to fulfill both constraints and serve the 1000 users that are communicating simultaneously, 33 three sector antenna BSs are placed as it is shown in Fig. 3. We can clearly see that the number of BSs placed in each subarea is proportional to the corresponding user density: around 11 and $7 \mathrm{BSs}$ are placed in subarea 1 and subarea 2, respectively, while 4 and 11 are deployed in subarea 3 and subarea 4 . Subarea 2 presents the highest user density with 24 users $/ \mathrm{km}^{2}$ while the density in subarea 3 is only 3 users $/ \mathrm{km}^{2}$. Note that subarea 3 requires only 4 BSs to cover its surface and serve its users whereas $7 \mathrm{BSs}$ are needed to satisfy the user density constraint in subarea 2 . However, for subarea 4, the number of BSs is mainly related to the coverage constraint since according to the capacity dimensioning phase in this subarea $3 \mathrm{BSs}$ are enough to serve the 200 users in that area. The Monte Carlo simulation indicates that the percentage of users in outage is around $0.21 \%$ and $0.26 \%$ for PSO and GWO, respectively, which respects the desired QoS. This proves the efficiency of the proposed practical meta-heuristic methods. We notice that both algorithms provide almost the same BS locations with minor differences. However, we can see through Fig. 4 that PSO algorithm is faster than GWO in terms of convergence speed. Indeed, GWO requires an exploration phase before it starts converging to its solutions which might require a relatively important number of iterations. Furthermore, by experiments and for large number of realizations, PSO algorithm is able to achieve easily the target utility for $\tau=98 \%$ while GWO might miss the target for around $20 \%$ of the realizations. In other words, the convergence rate of PSO in cell planning is significantly higher than that of GWO algorithm.

PSO and GWO are two meta-heuristic algorithms where the exact number of iterations needed to reach the solution is arbitrary and depends on the studied scenario. However, 


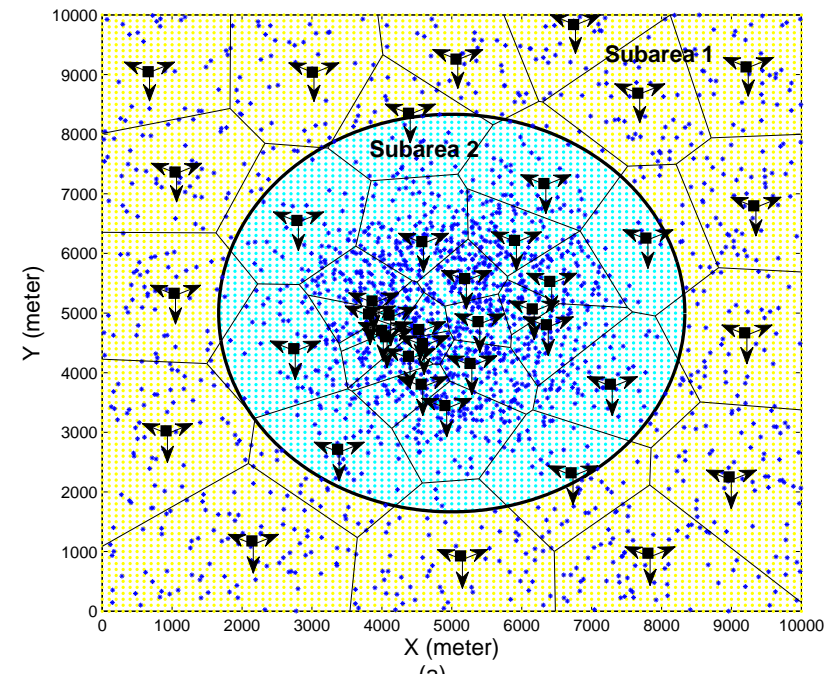

(a)

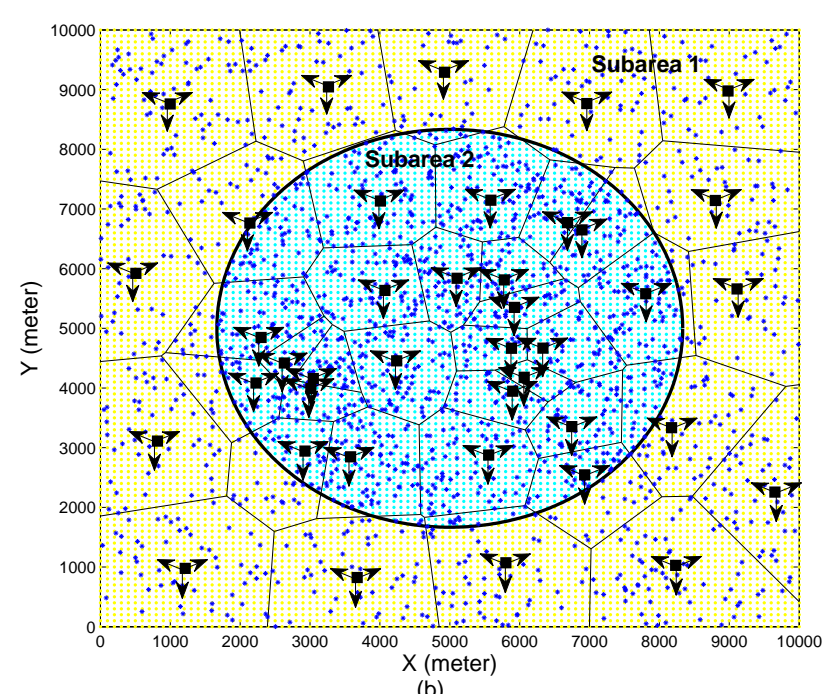

Fig. 2: Comparison between (a) Scenario A and (b) Scenario B using PSO algorithm (BS: square, MS: dot, BS sector:

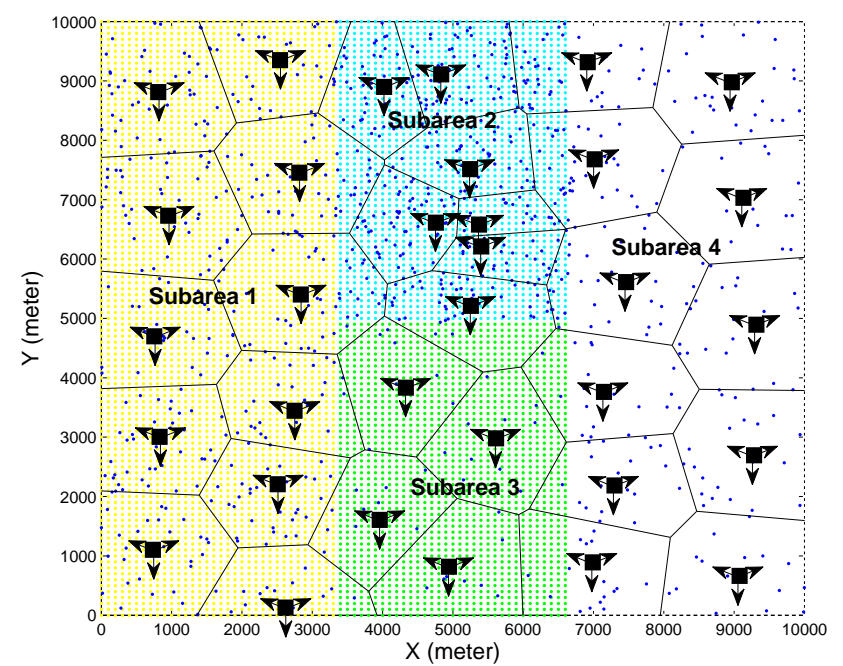

(a)

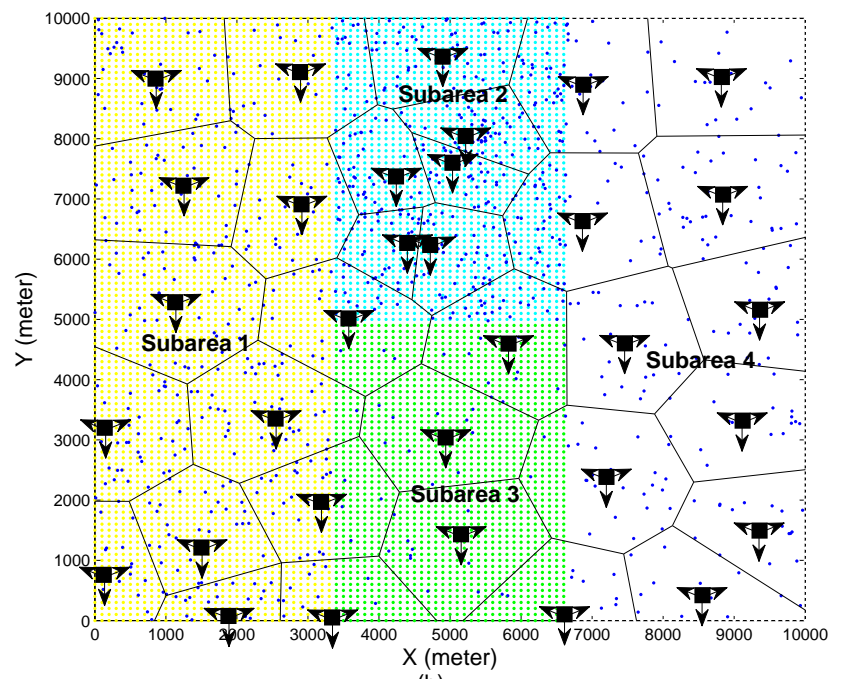

(b)

Fig. 3: Comparison between (a) PSO and (b) GWO for Scenario C (BS: square, MS: dot, BS sector: arrow).

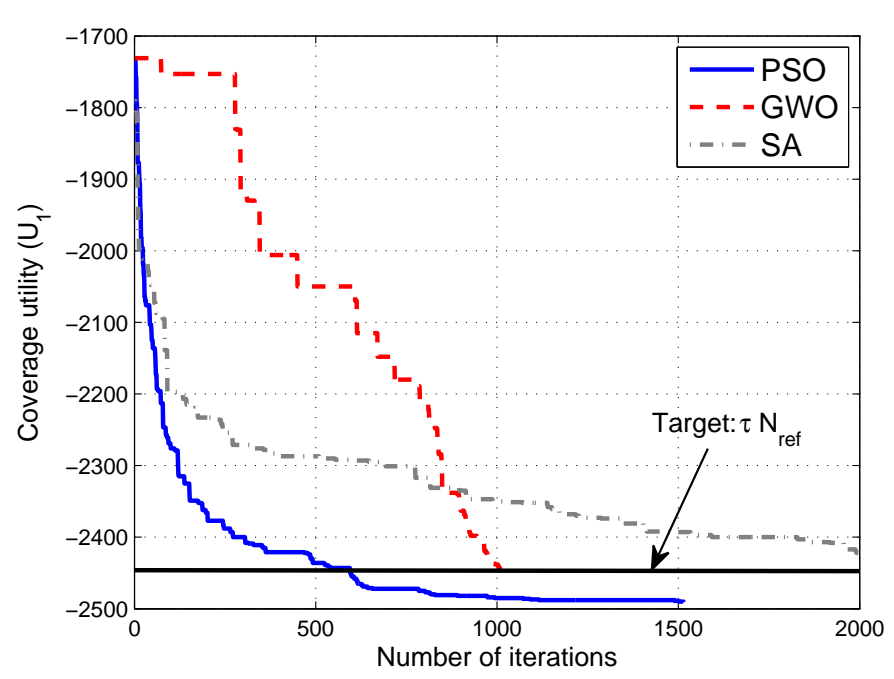

Fig. 4: Convergence speed with $L=12$.

the computational complexity per iteration can be determined. According to (16) and (17), PSO needs to calculate 10 multiplications and 10 additions for every BS position $\left(x_{j}(t), y_{j}(t)\right)$. Hence, $10 L N_{\mathrm{BS}}$ multiplications and $10 L N_{\mathrm{BS}}$ additions are calculated every iteration whereas GWO calculates $13 L N_{\mathrm{BS}}$ multiplications and $11 L N_{\mathrm{BS}}$ additions according to (18) and (19). In our simulation results, we assumed that both algorithms are executed at most 2000 iterations and are stopped if coverage and cell capacity constraints are satisfied $\tau=98 \%$. Thus, the utilities are computed at most $2000 \times L$ times. For 200 realizations, $L=\{12,24\}$ and $N_{\mathrm{BS}}=33$ and for Scenario $C$, results show that on average PSO is faster than GWO and requires less time to converge as it is shown in Table IV; in Table IV, we compute the CPU time in seconds of all algorithms and record the iteration where the algorithm satisfies both constraints (denoted as $I^{*}$ ). Increasing the number of particles $L$ would enhance the convergence speed of the algorithms. In fact, PSO and GWO are able to achieve their solutions with a lower number of iterations but they require more CPU time as they need to perform more operations during each iteration. For instance, on average with $L=12$, PSO performs 67800 multiplications while with $L=24$, it needs 97680 multiplications to converge. Similar remarks can be noticed for GWO.

We also compare the performance of the proposed metaheuristic approaches with SA [15]. In the traditional SA, one 
BS is perturbed at each iteration. In our simulations, in order to enhance the speed of SA, we select a random integer $N$ between 1 and $N_{\mathrm{BS}}$, and then randomly we select $N$ out of the $N_{\text {BS }}$ BSs to update their locations, then we move each of these $N$ BSs by a random perturbation that does not exceed $V_{\max }$. Results show that SA presents similar convergence speed as PSO in the beginning but faces difficulties to achieve the target as the number of iterations increases. Then, its convergence speed becomes very slow as it is shown in Fig. 4. On the other hand, it is considered faster than PSO and GWO in terms of CPU time as it performs 2000 iterations in 415 seconds. This is due to the fact that $\mathrm{SA}$ requires on average $N_{\mathrm{BS}}$ additions and $N_{\mathrm{BS}}$ multiplications per iteration only. Note that all tests were performed on a desktop machine featuring an Intel Xeon CPU and running Windows 7 Professional. The clock of the machine is set to $2.66 \mathrm{GHz}$ with a $48 \mathrm{~GB}$ memory.

TABLE IV: Measured CPU running time in seconds for the various considered algorithms

\begin{tabular}{|c||c|c|}
\hline & CPU time & $I^{*}$ \\
\hline \hline PSO $(L=12)$ & 255 & 565 \\
\hline PSO $(L=24)$ & 344 & 407 \\
\hline GWO $(L=12)$ & 368 & 986 \\
\hline GWO $(L=24)$ & 516 & 821 \\
\hline SA & 415 & 2000 \\
\hline
\end{tabular}

\section{LTE Networks with Femtocell Access Points}

We extend the implementation of the proposed planning approach to take into account the presence of femtocell access points (FAPs) that can offload some amount of the traffic from the cellular network. We consider Scenario $C$ and place 100 FAPs uniformly over the area. The maximum range of a FAP is set to be 10 meters and each FAP is assumed to simultaneously serve at maximum 10 subscribers of the cellular network. We execute the proposed approach using the PSO algorithm. In this case, the FAPs are considered already deployed and the problem consists in finding the

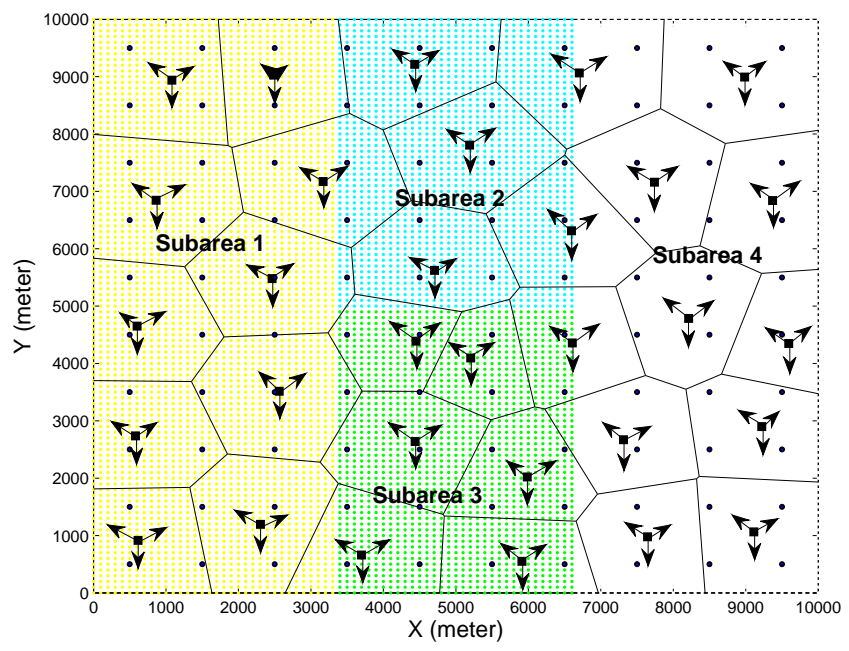

Fig. 5: LTE network scenario with femtocell access points: (BS: square, FAP: circle, BS sector: arrow).
BS locations taking into account the contribution of FAPs in serving users. Fig. 5 plots the positions of BSs using the proposed cell planning approach. It shows that $31 \mathrm{BSs}$ are deployed instead of 33 as it is given in Fig. 3(a). Indeed, in low density subareas, the number of BSs is the same for both cases, since it corresponds to the number of BSs needed to maintain the coverage constraint per each subarea; with a 10 meter range, FAP do not provide any enhancement in terms of total coverage. On the other hand, the number of BSs in subarea 2 is reduced and two BSs are eliminated as their presence is compensated by the existence of FAPs which can offload some users in that subarea.

\section{E. LTE Heterogeneous Networks}

We propose to apply our proposed planning approach for two-tier hetnet networks where macrocell BSs and small cell BSs co-exist. In this approach, we propose to:

(i) first apply the planning approach described in Section III for tri-sector macrocell BSs only until satisfying coverage and cell capacity constraints.

(ii) Then, we apply the planning approach considering small cell BSs equipped with omnidirectional antennas (antenna gain $12 \mathrm{dBi}$ ) only till satisfying the cell capacity constraint per each subarea. In fact, it is inefficient to deploy small cell BSs to ensure connectivity over the area especially in low density and rural zones due to their low coverage range (e.g., 260 meters in our case).

(iii) Finally, we place all BSs obtained from (i) and (ii) and apply the BS elimination algorithm in order to remove all redundant $\mathrm{BSs}$.

In Fig. 6, we show the obtained BS locations using the approach described above. 2000 users are distributed following a bimodal distribution consisting of a mixture of two truncated normal distributions centered in the points $(4 \mathrm{~km}, 7 \mathrm{~km})$ and $(7 \mathrm{~km}, 2.5 \mathrm{~km}$ ) with variances $1.3 \mathrm{~km}$ and $1 \mathrm{~km}$, respectively. The weight is set to 0.6 . We can clearly see, that in the rural subarea where only $7 \%$ of the users exist, only macrocell BSs are deployed to satisfy the coverage constraints and all the

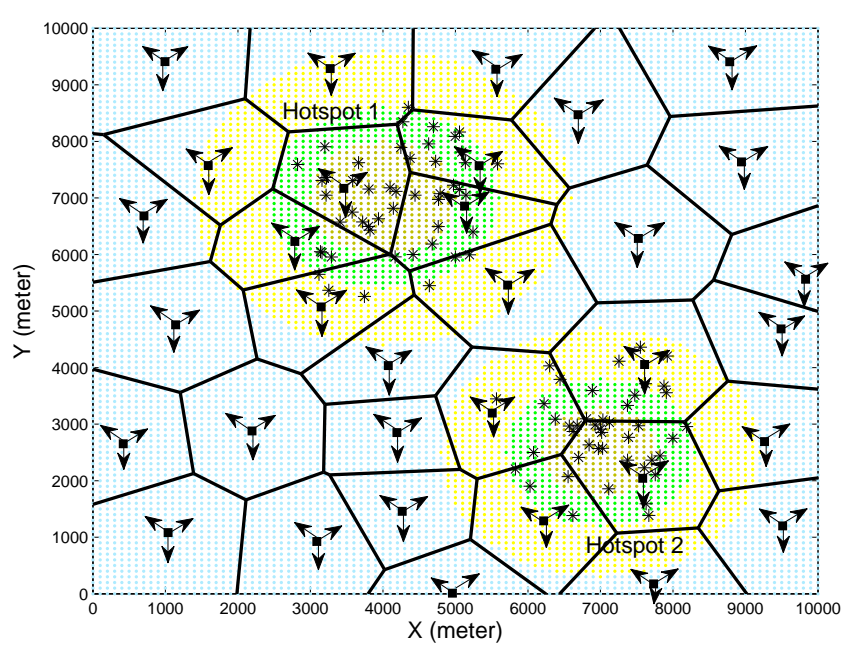

Fig. 6: LTE hetnet network scenario: (Macrocell BS: square, small cell BS: star, BS sector: arrow). 
small cell BSs are eliminated while in high density subarea macrocell BSs are placed and small cell BSs are deployed to fulfill the cell capacity constraints. Notice that the proposed approach adapted the BSs distribution to the given user traffic distribution as there is an important concentration of small cell BSs in both hotspot areas. These small cell BSs could be switched on/off depending on traffic load variation in order to reduce the overall network energy consumption.

\section{F. Green Planning}

In this problem, we consider Scenario A by assuming that 200 users are connecting at night while during day we assume that the number of users increases to 2000. During the night planning phase, we can see in Fig. 7 that the number of deployed BSs corresponds to the number of BSs required to ensure full coverage as the number of users is very low during this non-peak period. However, during the day, we can see that the BSs that are already deployed in subarea 1 are enough to serve the $40 \%$ of total users whereas the mobile operator needs to activate 14 BSs placed around the center of the Gaussian hotspot in order to satisfy the QoS of the subscribers.

\section{G. Planning with Location Constraints}

In order to demonstrate the performance of the proposed method presented in Section III, we consider a typical uniform user distribution over the area and we consider that placing BSs is prohibited in the central area (colored in red in Fig. 8(a)) of size $4 \times 4 \mathrm{~km}^{2}$ where the received power level at each point in that area has to be less than $-60 \mathrm{dBm}$. We can clearly see that the proposed method converges to the case where the BS placement follows the input user distribution while respecting the electromagnetic radiation constraint by locating the $27 \mathrm{BSs}$ outside the radiation free zone as it is shown in Fig. 8(b). We can see the difference with the nonlocation constraint problem where the $30 \mathrm{BSs}$ are allowed to be placed anywhere in the area and almost having the same locations as the black BSs mainly in the area boundaries.

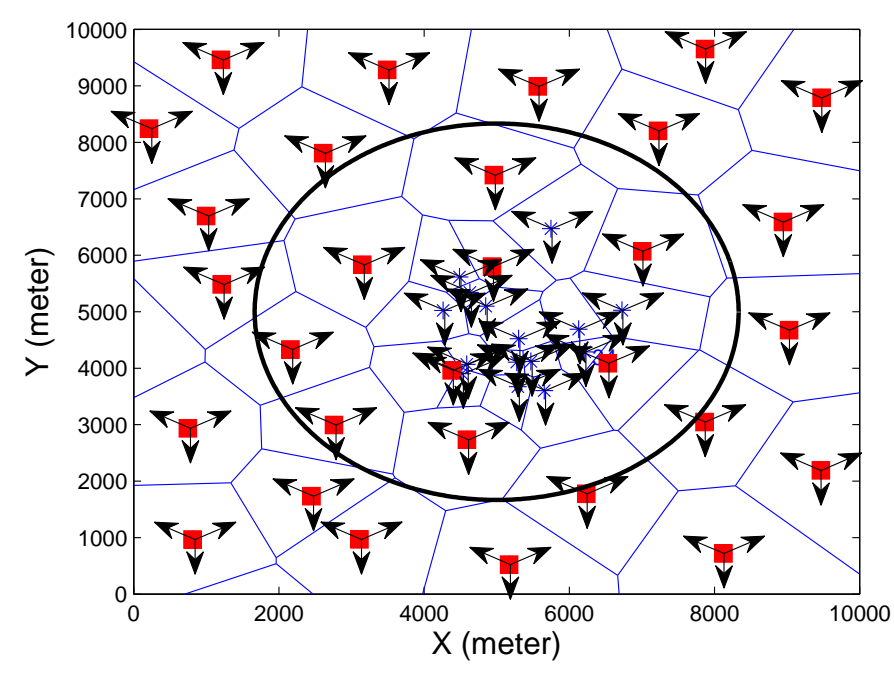

Fig. 7: Green planning: Night BSs (red squares), additional day BSs (blue stars).

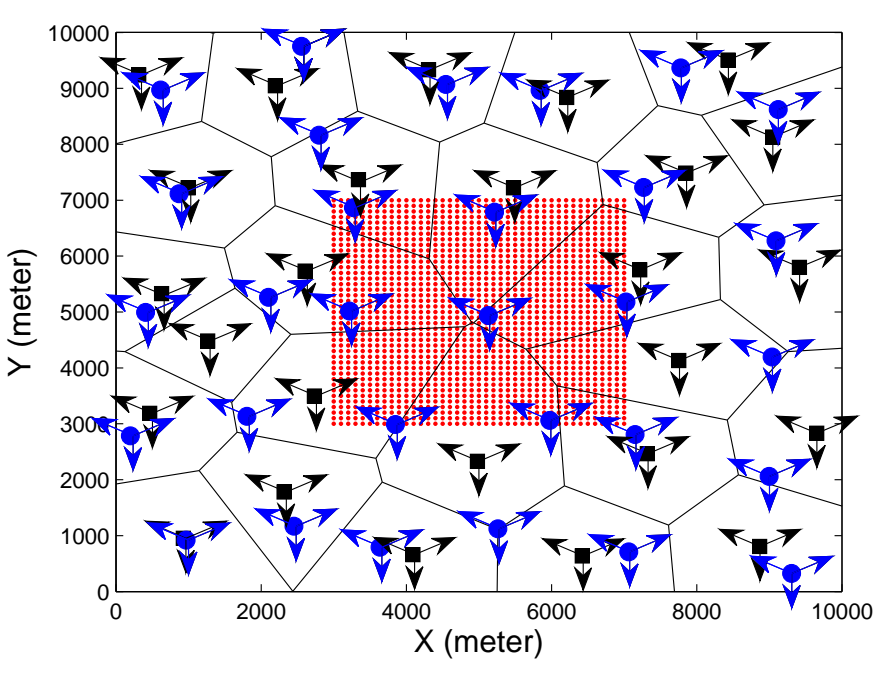

(a) Voronoi constellation, radiation-limited area in red (black squares: BSs with location constraints, blue circles: BSs without location

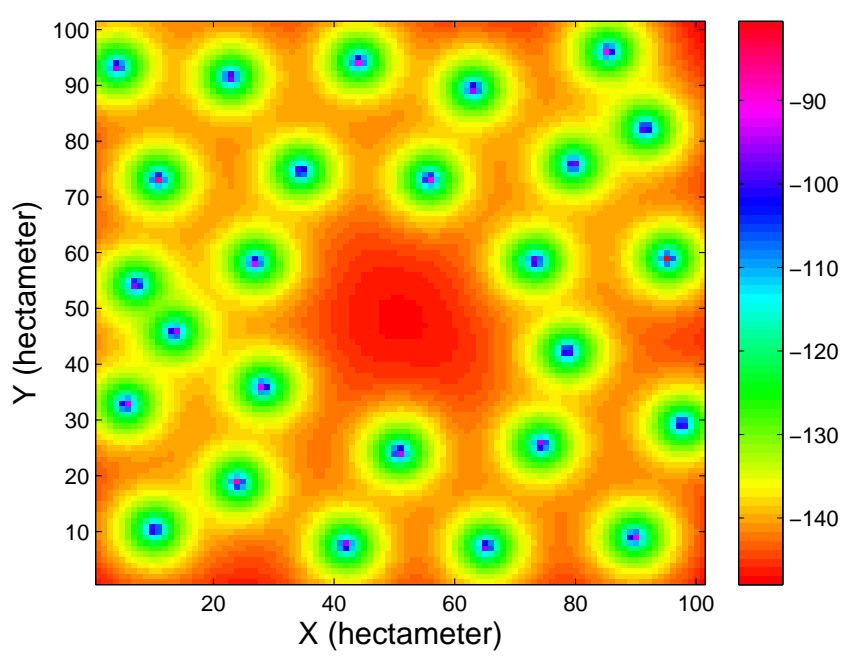

(b) Electromagnetic radiation levels in $\mathrm{dBm}$ over the area.

Fig. 8: BS placement for location constraint problem.

\section{ACKNOWLEDGMENT}

The authors would like to thank the editor and the anonymous reviewers for their valuable comments and suggestions that helped in enhancing the quality of the paper.

\section{CONCLUSION}

In this paper, we proposed an efficient planning method for 4G-LTE networks in order to deploy base stations while respecting two major constraints: the coverage and cell capacity constraints by taking into account several subareas characterized by different user densities, uplink and downlink directions, LTE resource allocation, and intercell interference. The proposed approach starts by performing a typical coverage and dimensioning phase. Then, it employs a meta-heuristic algorithm to find the optimal BS locations that fulfill the system constraints. Finally, it eliminates eventual redundant base stations to keep the minimum number of base stations required to ensure a safe network operation. Using Monte Carlo simulations, we have investigated the performance of our proposed 
scheme where we computed the average number of users in outage. We showed that it provides a very low outage rate and respects the desired network quality of service. Furthermore, we showed that the PSO algorithm outperforms the GWO algorithm in terms of convergence speed and convergence rate. Finally, we adapted our proposed method to perform a green planning that considers day/night traffic variation in order to provide energy savings and a planning with location constraint problem due to electromagnetic radiation limitation. We believe that the proposed $4 \mathrm{G}$ cell planning approach will be also useful for the development of planning algorithms for $5 \mathrm{G}$ networks, especially that we capture emerging aspects such as dynamic load variations, green considerations, heterogeneous networks and femtocell deployments.

\section{REFERENCES}

[1] H. Ghazzai, E. Yaacoub, and M.-S. Alouini, "Optimized LTE cell planning for multiple user density subareas using meta-heuristic algorithms," in Proc. of the 80th IEEE Vehicular Technology Conference (VTC Fall 2014), Vancouver, Canada, Sept. 2014.

[2] T. Bauschert, C. Busing, F. D’Andreagiovanni, A. Koster, M. Kutschka, and U. Steglich, "Network planning under demand uncertainty with robust optimization," IEEE Communications Magazine, vol. 52, no. 2, pp. 178-185, Feb. 2014.

[3] S. Boiardi, A. Capone, and B. Sanso, "Planning for energy-aware wireless networks," IEEE Communications Magazine, vol. 52, no. 2, pp. 156-162, Feb. 2014

[4] A. Imran, E. Yaacoub, Z. Dawy, and A. Abu-Dayya, "Planning future cellular networks: A generic framework for performance quantification," in Proc. of the 19th European Wireless Conference (EW 2013), Guildford, UK, Apr. 2013.

[5] E. Amaldi, A. Capone, and F. Malucelli, "Planning UMTS base station location: Optimization models with power control and algorithms," IEEE Transactions on Wireless Communications, vol. 2, no. 5, pp. 939-952, Sept. 2003.

[6] L. Shangyun and M. St-Hilaire, "A genetic algorithm for the global planning problem of UMTS networks," in Proc. of IEEE Global Telecommunications Conference (GLOBECOM 2010), Miami, FL, USA, Dec. 2010.

[7] C. Lee and H. Kang, "Cell planning with capacity expansion in mobile communications: A TABU search approach," IEEE Transactions on Vehicular Technology, vol. 49, no. 5, pp. 1678-1691, Sept. 2000.

[8] F. Gordejuela-Sanchez and J. Zhang, "LTE access network planning and optimization: A service-oriented and technology-specific perspective," in Proc. of IEEE Global Telecommunications Conference (GLOBECOM 2009), Honolulu, HI, USA, Dec. 2009.

[9] A. Guo and M. Haenggi, "Spatial stochastic models and metrics for the structure of base stations in cellular networks," IEEE Transactions on Wireless Communications, vol. 12, no. 11, pp. 5800-5812, Nov. 2013.

[10] A. Abdel Khalek, L. Al-Kanj, Z. Dawy, and G. Turkiyyah, "Optimization models and algorithms for joint uplink/downlink UMTS radio network planning with SIR-Based power control," IEEE Transactions on Vehicular Technology, vol. 60, no. 4, no. 4, pp. 1612-1625, May 2011.

[11] W. El-Beaino, A. M. El-Hajj, and Z. Dawy, "A proactive approach for LTE radio network planning with Green considerations," in Proc. of the 19th IEEE International Conference on Telecommunications (ICT 2012), Jounieh, Lebanon, Apr. 2012.

[12] M. Zheng, P. Pawelczak, S. Stanczak, and H. Yu, "Planning of cellular networks enhanced by energy harvesting," IEEE Communications Letters, vol. 17, no. 6, pp. 1092-1095, Jun. 2013.

[13] J. Kennedy and R. Eberhart, "Particle swarm optimization," in Proc. of IEEE International Conference on Neural Networks, Perth, Australia, Nov. 1995.

[14] S. Mirjalili, S-M. Mirjalili, and A. Lewis, "Grey wolf optimizer," Elsevier Advances in Engineering Software, vol. 69, no. 1, pp. 46-61, Mar. 2014.

[15] S. Kirkpatrick, C. D. Jr. Gerlatt, and M.P. Vecchi, "Optimization by simulated annealing," Science, vol. 220, no. 4598, pp. 671-680, 1983.

[16] H.Holma and A.Toskala, WCDMA for UMTS: HSPA Evolution and LTE. John Wiley \& Sons, 2010.

[17] H.Holma and A.Toskala, LTE for UMTS: OFDMA and SC-FDMA Based Radio Access. John Wiley \& Sons, 2009.
[18] C. Johnson, Long term evolution in bullets, 2nd edition. Northhampton, UK: CreateSpace, 2012.

[19] COST Action 231, "Digital Mobile Radio Towards Future Generation Systems," tech. rep., European Communities, EUR 18957, 1999.

[20] D. E. Goldberg, Genetic Algorithms in Search, Optimization and Machine Learning. Reading, MA, USA: Addison-Wesley, 1989.

[21] M. Dorigo, M. Birattari, and T. Stutzle, "Ant colony optimization," IEEE Computational Intelligence Magazine, vol. 1, no. 4, pp. 28-39, Nov. 2006.

[22] M. Clerc and J. Kennedy, "The particle swarm-explosion, stability, and convergence in a multidimensional complex space," IEEE Transactions on Evolutionary Computation, vol. 6, no. 3, pp. 58-73, Feb. 2002.

[23] H. Chen, C. Tse, and J. Feng, "Minimizing effective energy consumption in multi-cluster sensor networks for source extraction," IEEE Transactions on Wireless Communications, vol. 8, no. 3, pp. 1480-1489, Mar. 2009.

[24] J. Zhang, S. Chen, X. Mu, and L. Hanzo, "Evolutionary-algorithmassisted joint channel estimation and turbo multiuser detection/decoding for OFDM/SDMA," IEEE Transactions on Vehicular Technology, vol. 63, no. 3, pp. 1204-1222, Mar. 2014.

[25] S. Efazati and P. Azmi, "Effective capacity maximization in multirelay networks with a novel cross-layer transmission framework and powerallocation scheme," IEEE Transactions on Vehicular Technology, vol. 63, no. 4, pp. 1691-1702, May 2014.

[26] Q. Bai, "Analysis of particle swarm optimization algorithm," Computer and Information Science, vol. 3, no. 1, Feb. 2010.

[27] IEEE Standard C95. 1, "IEEEs standard for safety levels with respect to human exposure to radio frequency electromagnetic fields, $3 \mathrm{kHz}$ to 300 GHz," Mar. 2012.

[28] M. Mirahmadi, A. Al-Dweik, and A. Shami, "BER reduction of OFDM based broadband communication systems over multipath channels with impulsive noise," IEEE Transactions on Communications, vol. 61, pp. 4602-4615, Nov. 2013.

[29] D. Dechene and A. Shami, "Energy-aware resource allocation strategies for LTE uplink with synchronous HARQ constraints," IEEE Transactions on Mobile Computing, vol. 13, pp. 422-433, Feb. 2014.

[30] M. Mirahmadi, A. Al-Dweik, and A. Shami, "Interference modeling and performance evaluation of heterogeneous cellular networks," IEEE Transactions on Communications, vol. 62, pp. 2132-2144, Jun. 2014.

[31] I. Viering, M. Dottling, and A. Lobinger, "A mathematical perspective of self-optimizing wireless networks," in IEEE International Conference on Communications (ICC 2009), Dresden, Germany, Jun. 2009.

[32] A. Imran, M. Imran, and R. Tafazolli, "Relay station access link spectral efficiency optimization through SO of macro BS tilts," IEEE Communications Letters, vol. 15, no. 2, pp. 1326-1328, Dec. 2011.

[33] J. Lim, H.G. Myung, K. Oh, and D.J. Goodman, "Channel-dependent scheduling of uplink single carrier FDMA systems," in Proc. of IEEE Vehicular Technology Conference (VTC-Fall 2006), Montreal, Canada, Sept. 2006

[34] T. Lunttila, J. Lindholm, K. Pajukoski, E. Tiirola, and A. Toskala, "EUTRAN uplink performance," in Proc. of International Symposium on Wireless Pervasive Computing (ISWPC 2007), San Juan, Puerto Rico, Feb. 2007.

[35] H. G. Myung and D. J. Goodman, Single Carrier FDMA: A New Air Interface for Long Term Evolution. Wiley, 2008.

[36] Y. Huang and B. Rao, "An analytical framework for heterogeneous partial feedback design in heterogeneous multicell OFDMA networks," IEEE Transactions on Signal Processing, vol. 61, no. 3, pp. 753-769, Feb. 2013.

[37] 3rd Generation Partnership Project (3GPP), "3GPP TS 36.213 3GPP TSG RAN Evolved Universal Terrestrial Radio Access (E-UTRA) Physical Layer Procedures, version 11.4.0, Release 11," 2013.

[38] 3rd Generation Partnership Project (3GPP), "3GPP TS 36.211 3GPP TSG RAN Evolved Universal Terrestrial Radio Access (E-UTRA) Physical Channels and Modulation, version 11.4.0, Release 11," 2013. 


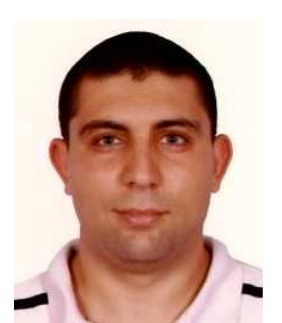

Hakim Ghazzai (S12, M15) was born in Tunisia. He received his $\mathrm{PhD}$ degree in Electrical Engineering from King Abdullah University of Science and Technology (KAUST) in Saudi Arabia in 2015. He received his Diplome d'Ingenieur in telecommunication engineering with highest distinction from the Ecole Superieure des Communications de Tunis (SUP'COM), Tunis, Tunisia in 2010 and his Master degree in High-Rate Transmission Systems from the same institute in 2011. His general research interests communications and optimization.

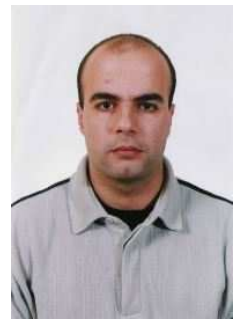

Elias Yaacoub (S07, M10, SM14) received the B.E. degree in Electrical Engineering from the Lebanese University in 2002, the M.E. degree in Computer and Communications Engineering from the American University of Beirut (AUB) in 2005, and the PhD degree in Electrical and Computer Engineering from AUB in 2010. He worked as a Research Assistant in the American University of Beirut from 2004 to 2005, and in the Munich University of Technology in Spring 2005. From 2005 to 2007, he worked as a Telecommunications Engineer with Dar AlHandasah, Shair and Partners. From 2010 to 2014, he was a Research Scientist at the Qatar Mobility Innovations Center (QMIC), where he led the broadband wireless access group since July 2012. He led and participated in several multinational research projects on topics related to $4 \mathrm{G} / 5 \mathrm{G}$ cellular systems, connectivity in vehicular and railroad networks, video transmission over wireless networks, sensor networks, internet of things, smart grid communications, in addition to air quality monitoring and data analysis. He is currently a consultant with Strategic Decisions Group (SDG).

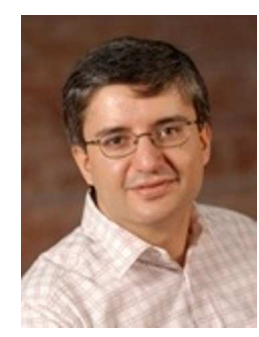

Mohamed-Slim Alouini (S'94, M'98, SM'03, F09) was born in Tunis, Tunisia. He received the Ph.D. degree in Electrical Engineering from the California Institute of Technology (Caltech), Pasadena, CA, USA, in 1998. He served as a faculty member in the University of Minnesota, Minneapolis, MN, USA, then in the Texas A\&M University at Qatar, Education City, Doha, Qatar before joining King Abdullah University of Science and Technology (KAUST), Thuwal, Makkah Province, Saudi Arabia as a Professor of Electrical Engineering in 2009. His current research interests include the modeling, design, and performance analysis of wireless communication systems.

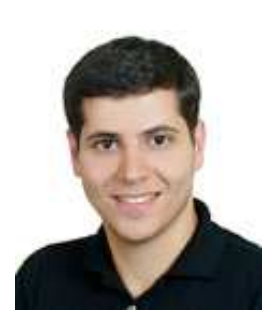

Zaher Dawy (SM09) received the B.E. degree in computer and communications engineering from the American University of Beirut (AUB), Beirut, Lebanon, in 1998 and the M.E. and Dr.-Ing. degrees in communications engineering from Munich University of Technology (TUM), Munich, Germany, in 2000 and 2004, respectively. Since September 2004, he has been with AUB, where he is currently a Professor of Electrical and Computer Engineering. His research and teaching interests include distributed and cooperative communications, cellular technologies, context-aware mobile computing, mobile solutions for smart cities, computational genomics, and biomedical engineering. Dr. Dawy currently serves on the editorial board for IEEE Transactions on Communications, IEEE Transactions on Wireless Communications, IEEE Communications Surveys and Tutorials, Elsevier Physical Communications, and Elsevier Pervasive and Mobile Computing. Before, he served as an Executive Editor for Wiley Transactions on Emerging Telecommunications Technologies and as the Chair of the IEEE Communications Society Lebanon Chapter. He is the recipient of the Abdul Hameed Shoman Award for Young Arab Researchers in 2012, IEEE Communications Society 2011 Outstanding Young Researcher Award in Europe, Middle East, and Africa Region, AUB Teaching Excellence Award in 2008, Best MSCE Graduate Award from TUM in 2000, Youth and Knowledge Siemens Scholarship for Distinguished Students in 1999, and Distinguished Graduate Medal of Excellence from Hariri Foundation in 1998.

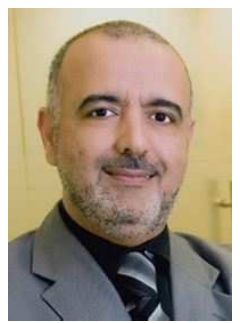

Adnan Abu-Dayya received his $\mathrm{PhD}$ in Electrical Engineering from Queens University, Canada in 1992. He then worked as a manager at Nortel Networks in Canada in the advanced technology group and as a Senior Consultant at the Communications Research Center in Ottawa, Canada. Before moving to Qatar in March 2007, he worked for 10 years at AT\&T Wireless in Seattle, USA where he served in a number of senior management positions covering product innovations and emerging technologies, systems engineering, and product realization. He was also responsible for developing and licensing the extensive patent portfolio of AT\&T Wireless. From April 2007 to December 2008, he was the chairman of the Electrical Engineering Department at Qatar University (QU). He was appointed as the Executive Director of the QU Wireless Innovations Center (QUWIC) in December 2008 (QUWIC was rebranded to QMIC (Qatar Mobility Innovations Center) in July 2012). Dr. Abu-Dayya has more than 20 years of International experience in the areas of wireless/telecomm R\&D, innovations, business development, and services delivery. He has many issued patents and more than fifty publications in the field of wireless communications. 